PAPER • OPEN ACCESS

\title{
Performance of the MICE diagnostic system
}

To cite this article: The MICE collaboration et al 2021 JINST 16 P08046

View the article online for updates and enhancements.

The Electrochemical Society 2021 Virtual Education

\section{Intensive Short Courses}

Sunday, October 10 \& Monday, October 11

Providing students and professionals with in-depth education on a wide range of topics

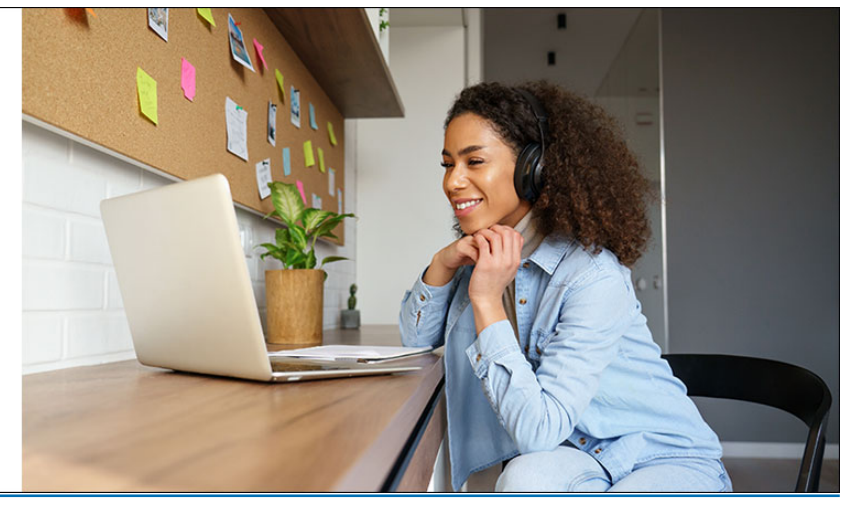

\section{CLICK HERE TO REGISTER}




\section{Performance of the MICE diagnostic system}

\section{The MICE collaboration}
M. Bogomilov, ${ }^{a}$ R. Tsenov, ${ }^{a}$ G. Vankova-Kirilova, ${ }^{a}$ Y.P. Song, ${ }^{b}$ J.Y. Tang, ${ }^{b}$ Z.H. Li, ${ }^{c}$
R. Bertoni, ${ }^{d}$ M. Bonesini, ${ }^{d}$ F. Chignoli, ${ }^{d}$ R. Mazza, ${ }^{d}$ V. Palladino, ${ }^{e}$ A. de Bari, ${ }^{f}$ D. Orestano, ${ }^{g}$
L. Tortora, ${ }^{g}$ Y. Kuno, ${ }^{h}$ H. Sakamoto, ${ }^{h, 1}$ A. Sato, ${ }^{h}$ S. Ishimoto,${ }^{i}$ M. Chung,${ }^{j}$ C.K. Sung, ${ }^{j}$
F. Filthaut, ${ }^{k, 2}$ M. Fedorov, ${ }^{l}$ D. Jokovic, ${ }^{m}$ D. Maletic, ${ }^{m}$ M. Savic, ${ }^{m}$ N. Jovancevic, ${ }^{n}$ J. Nikolov, ${ }^{n}$
M. Vretenar, ${ }^{o}$ S. Ramberger, ${ }^{o}$ R. Asfandiyarov, ${ }^{p}$ A. Blondel, ${ }^{p}$ F. Drielsma, ${ }^{p, 3}$ Y. Karadzhov, ${ }^{p}$
G. Charnley, ${ }^{q}$ N. Collomb, ${ }^{q}$ K. Dumbell, ${ }^{q}$ A. Gallagher, ${ }^{q}$ A. Grant, ${ }^{q}$ S. Griffiths, ${ }^{q}$ T. Hartnett, ${ }^{q}$
B. Martlew, ${ }^{q}$ A. Moss, ${ }^{q}$ A. Muir, ${ }^{q}$ I. Mullacrane, ${ }^{q}$ A. Oates, ${ }^{q}$ P. Owens, ${ }^{q}$ G. Stokes, ${ }^{q}$
P. Warburton, ${ }^{q}$ C. White, ${ }^{q}$ D. Adams, ${ }^{r}$ V. Bayliss, ${ }^{r}$ J. Boehm, ${ }^{r}$ T.W. Bradshaw, ${ }^{r}$ C. Brown, ${ }^{r, 4}$
M. Courthold ${ }^{r}$ J. Govans, ${ }^{r}$ M. Hills, ${ }^{r}$ J.-B. Lagrange, ${ }^{r}$ C. Macwaters, ${ }^{r}$ A. Nichols, ${ }^{r}$
R. Preece, ${ }^{r}$ S. Ricciardi, ${ }^{r}$ C. Rogers, ${ }^{r}$ T. Stanley, ${ }^{r}$ J. Tarrant, ${ }^{r}$ M. Tucker, ${ }^{r}$ S. Watson, ${ }^{r}, 5$
A. Wilson, ${ }^{r}$ R. Bayes, ${ }^{s, 6}$ J.C. Nugent, ${ }^{s}$ F.J.P. Soler, ${ }^{s}$ R. Gamet, ${ }^{t}$ P. Cooke,${ }^{t}$ V.J. Blackmore, ${ }^{u}$
D. Colling, ${ }^{u}$ A. Dobbs, ${ }^{u}, 7$ P. Dornan, ${ }^{u}$ P. Franchini, ${ }^{u, *}$ C. Hunt,,${ }^{u, 8}$ P.B. Jurj, ${ }^{u}$ A. Kurup, ${ }^{u}$
K. Long, ${ }^{u}$ J. Martyniak, ${ }^{u}$ S. Middleton, ${ }^{u}, 9$ J. Pasternak, ${ }^{u}$ M.A. Uchida, ${ }^{u}, 10$ J.H. Cobb, ${ }^{v}$
C.N. Booth, ${ }^{w}$ P. Hodgson, ${ }^{w}$ J. Langlands, ${ }^{w}$ E. Overton, ${ }^{w}, 11$ V. Pec, ${ }^{w}$ P.J. Smith, ${ }^{w}$
S. Wilbur, ${ }^{w}$ G.T. Chatzitheodoridis, ${ }^{x, 12,13}$ A.J. Dick, ${ }^{x, 13}$ K. Ronald, ${ }^{x, 13}$ C.G. Whyte, ${ }^{x, 13}$

A.R. Young, ${ }^{x, 13}$ S. Boyd,${ }^{y}$ J.R. Greis, ${ }^{y, 14}$ T. Lord, ${ }^{y}$ C. Pidcott,,${ }^{y, 15}$ I. Taylor,,${ }^{y, 16}$ M. Ellis, ${ }^{z, 17}$

\footnotetext{
${ }^{1}$ Current address: RIKEN 2-1 Hirosawa, Wako, Saitama, Japan.

${ }^{2}$ Also at Radboud University, Houtlaan 4, Nijmegen, Netherlands.

${ }^{3}$ Current address: SLAC National Accelerator Laboratory, 2575 Sand Hill Road, Menlo Park, CA, U.S.A.

${ }^{4}$ Also at College of Engineering, Design and Physical Sciences, Brunel University, Kingston Lane, Uxbridge, U.K.

${ }^{5}$ Current address: ATC, Royal Observatory Edinburgh, Blackford Hill, Edinburgh, U.K.

${ }^{6}$ Current address: Laurentian University, 935 Ramsey Lake Road, Sudbury, ON, Canada.

${ }^{7}$ Current address: OPERA Simulation Software, Network House, Langford Locks, Kidlington, U.K.

${ }^{8}$ Current address: CERN, Esplanade des Particules 1, Geneva, Switzerland.

${ }^{9}$ Current address: School of Physics and Astronomy, University of Manchester, Oxford Road, Manchester, U.K.

${ }^{10}$ Current address: Rutherford Building, Cavendish Laboratory, JJ Thomson Avenue, Cambridge, U.K.

${ }^{11}$ Current address: Arm, City Gate, 8 St Mary's Gate, Sheffield, U.K.

${ }^{12}$ Also at School of Physics and Astronomy, Kelvin Building, University of Glasgow, Glasgow, U.K.

${ }^{13}$ Also at Cockcroft Institute, Daresbury Laboratory, Sci-Tech Daresbury, Keckwick Ln, Daresbury, Warrington, U.K.

${ }^{14}$ Current address: TNG Technology Consulting, Beta-Strasse 13A, Unterföhring, Germany.

${ }^{15}$ Current address: Department of Physics and Astronomy, University of Sheffield, Hounsfield Rd, Sheffield, U.K.

${ }^{16}$ Current address: Defence Science and Technology Laboratory, Porton Down, Salisbury, U.K.

${ }^{17}$ Current address: Macquarie Group, 50 Martin Place, Sydney, Australia.
} 
R.B.S. Gardener, ${ }^{z, 18}$ P. Kyberd, ${ }^{z}$ J.J. Nebrensky, ${ }^{z}$ M. Palmer, ${ }^{a a}$ H. Witte, ${ }^{a a}$ D. Adey, ${ }^{a b, 19}$ A.D. Bross, ${ }^{a b}$ D. Bowring, ${ }^{a b}$ P. Hanlet, ${ }^{a b}$ A. Liu, ${ }^{a b, 20}$ D. Neuffer, ${ }^{a b}$ M. Popovic, ${ }^{a b}$ P. Rubinov, ${ }^{a b}$ A. DeMello, ${ }^{a c}$ S. Gourlay, ${ }^{a c}$ A. Lambert, ${ }^{a c}$ D. Li, ${ }^{a c}$ T. Luo, ${ }^{a c}$ S. Prestemon, ${ }^{a c}$ S. Virostek, ${ }^{a c}$ B. Freemire, ${ }^{a d, 20}$ D.M. Kaplan, ${ }^{a d}$ T.A. Mohayai, ${ }^{a d, 21}$ D. Rajaram, ${ }^{a d, 22}$ P. Snopok, ${ }^{a d}$ Y. Torun, ${ }^{a d}$ L.M. Cremaldi, ${ }^{a e}$ D.A. Sanders, ${ }^{a e}$ D.J. Summers, ${ }^{a e, 23}$ L.R. Coney, ${ }^{a f, 24}$ G.G. Hanson ${ }^{a f}$ and C. Heidt ${ }^{a f, 25}$

${ }^{a}$ Department of Atomic Physics, St. Kliment Ohridski University of Sofia, 5 James Bourchier Blvd, Sofia, Bulgaria

${ }^{b}$ Institute of High Energy Physics, Chinese Academy of Sciences, 19 Yuquan Rd, Shijingshan District, Beijing, China

${ }^{c}$ Sichuan University, 252 Shuncheng St, Chengdu, China

${ }^{d}$ Sezione INFN Milano Bicocca and Dipartimento di Fisica G. Occhialini, Piazza della Scienza 3, Milano, Italy

${ }^{e}$ Sezione INFN Napoli and Dipartimento di Fisica, Università Federico II, Complesso Universitario di Monte S. Angelo, Via Cintia, Napoli, Italy

${ }^{f}$ Sezione INFN Pavia and Dipartimento di Fisica, Università di Pavia, Via Agostino Bassi 6, Pavia, Italy

${ }^{g}$ Sezione INFN Roma Tre and Dipartimento di Matematica e Fisica, Università Roma Tre,

Via della Vasca Navale 84, Roma, Italy

${ }^{h}$ Osaka University, Graduate School of Science, Department of Physics,

1-1 Machikaneyamacho, Toyonaka, Osaka, Japan

${ }^{i}$ High Energy Accelerator Research Organization (KEK), Institute of Particle and Nuclear Studies, Tsukuba, Ibaraki, Japan

${ }^{j}$ Department of Physics, UNIST, 50 UNIST-gil, Ulsan, South Korea

${ }^{k}$ Nikhef, Science Park 105, Amsterdam, Netherlands

${ }^{l}$ Radboud University, Houtlaan 4, Nijmegen, Netherlands

${ }^{m}$ Institute of Physics, University of Belgrade, Pregrevica 118, Belgrade, Serbia

${ }^{n}$ Faculty of Sciences, University of Novi Sad, Trg Dositeja Obradovića 3, Novi Sad, Serbia

${ }^{o}$ CERN, Esplanade des Particules 1, Geneva, Switzerland

${ }^{p}$ DPNC, Section de Physique, Université de Genève, 24 Quai Ernest-Ansermet, Geneva, Switzerland

${ }^{q}$ STFC Daresbury Laboratory, Keckwick Ln, Daresbury, Cheshire, U.K.

${ }^{r}$ STFC Rutherford Appleton Laboratory, Harwell Campus, Didcot, U.K.

${ }^{s}$ School of Physics and Astronomy, Kelvin Building, University of Glasgow, Glasgow, U.K.

${ }^{t}$ Department of Physics, University of Liverpool, Oxford St, Liverpool, U.K.

${ }^{u}$ Department of Physics, Blackett Laboratory, Imperial College London, Exhibition Road, London, U.K.

${ }^{v}$ Department of Physics, University of Oxford, Denys Wilkinson Building, Keble Rd, Oxford, U.K.

${ }^{w}$ Department of Physics and Astronomy, University of Sheffield, Hounsfield Rd, Sheffield, U.K.

${ }^{18}$ Current address: Inawisdom, Columba House, Adastral park, Martlesham, Ipswich, U.K.

${ }^{19}$ Current address: Institute of High Energy Physics, Chinese Academy of Sciences, 19 Yuquan Rd, Shijingshan District, Bejing, China.

${ }^{20}$ Current address: Euclid Techlabs, 367 Remington Blvd, Bolingbrook, IL, U.S.A.

${ }^{21}$ Current address: Fermilab, Kirk Rd and Pine St, Batavia, IL, U.S.A.

${ }^{22}$ Current address: KLA, 2350 Green Rd, Ann Arbor, MI, U.S.A.

${ }^{23}$ Deceased.

${ }^{24}$ Current address: European Spallation Source ERIC, Partikelgatan 2, Lund, Sweden.

${ }^{25}$ Current address: Swish Analytics, Oakland, CA, U.S.A.

*Corresponding author. 
${ }^{x}$ SUPA and the Department of Physics, University of Strathclyde, 107 Rottenrow, Glasgow, U.K.

${ }^{y}$ Department of Physics, University of Warwick, Gibbet Hill Road, Coventry, U.K.

${ }^{z}$ College of Engineering, Design and Physical Sciences, Brunel University,

Kingston Lane, Uxbridge, U.K.

${ }^{a a}$ Brookhaven National Laboratory, 98 Rochester St, Upton, NY, U.S.A.

${ }^{a b}$ Fermilab, Kirk Rd and Pine St, Batavia, IL, U.S.A.

${ }^{a c}$ Lawrence Berkeley National Laboratory, 1 Cyclotron Rd, Berkeley, CA, U.S.A.

ad Illinois Institute of Technology, 10 West 35th St, Chicago, IL, U.S.A.

${ }^{a e}$ University of Mississippi, University Ave, Oxford, MS, U.S.A.

${ }^{\text {af }}$ University of California, 900 University Ave, Riverside, CA, U.S.A.

E-mail: p.franchini@imperial.ac.uk

ABSTRACT: Muon beams of low emittance provide the basis for the intense, well-characterised neutrino beams of a neutrino factory and for multi-TeV lepton-antilepton collisions at a muon collider. The international Muon Ionization Cooling Experiment (MICE) has demonstrated the principle of ionization cooling, the technique by which it is proposed to reduce the phase-space volume occupied by the muon beam at such facilities. This paper documents the performance of the detectors used in MICE to measure the muon-beam parameters, and the physical properties of the liquid hydrogen energy absorber during running.

Keywords: Accelerator Applications; Accelerator modelling and simulations (multi-particle dynamics, single-particle dynamics); Accelerator Subsystems and Technologies; Beam-line instrumentation (beam position and profile monitors, beam-intensity monitors, bunch length monitors)

ARXiv ePrint: 2106.05813 


\section{Contents}

1 Introduction 1

2 Time-of-flight detectors 3

3 Cherenkov detectors $\quad 5$

$\begin{array}{lll}4 & \text { KLOE-light calorimeter } & 7\end{array}$

5 Electron muon ranger 9

6 Tracking 11

$\begin{array}{lll}6.1 \text { Trackers } & 11\end{array}$

$\begin{array}{lll}6.2 & \text { Beam-based detector alignment } & 14\end{array}$

$\begin{array}{lll}7 & \text { Liquid hydrogen absorber } & 17\end{array}$

8 Summary and conclusions $\quad 21$

\section{Introduction}

Stored muon beams have been proposed as the basis of a facility capable of delivering leptonantilepton collisions at very high energy $[1,2]$ and as the source of uniquely well-characterised neutrino beams [3-5]. In the majority of designs for such facilities the muons are produced from the decay of pions created when an intense proton beam strikes a target. The phase-space volume occupied by the tertiary muon beam must be reduced (cooled) before the beam is accelerated and subsequently injected into a storage ring. The times taken to cool the beam using techniques that are presently in use at particle accelerators (synchrotron-radiation cooling [6], laser cooling [7-9], stochastic cooling [10], electron cooling [11] and frictional cooling [12]) are long when compared with the lifetime of the muon. Ionization cooling $[13,14]$, in which a muon beam is passed through a material (the absorber) where it loses energy, and is then re-accelerated, occurs on a timescale short compared with the muon lifetime. Ionization cooling is therefore the only technique available to cool the muon beam at a neutrino factory or muon collider. The international Muon Ionization Cooling Experiment (MICE) provided the proof-of-principle demonstration of the ionization-cooling technique [15].

MICE operated at the ISIS Neutron and Muon Source at the STFC Rutherford Appleton Laboratory. The ISIS synchrotron accelerates pulses of protons to a kinetic energy of $800 \mathrm{MeV}$ at $50 \mathrm{~Hz}$. For MICE operation, a titanium target was dipped into the halo of the proton beam at $0.78 \mathrm{~Hz}$. Pions created in the interaction of the beam and target were captured in a quadrupole triplet (see figure 1). A beam line composed of dipole, solenoid, and quadrupole magnets captured muons produced through pion decay and transported the resulting muon beam to the MICE apparatus. The 
momentum of the muon beam was determined by the settings of the two dipole magnets D1 and D2. Beams having muon central momenta between $140 \mathrm{MeV} / c$ and $240 \mathrm{MeV} / c$ were used for ionisation cooling studies. The emittance of the beam injected into the experiment was tuned using a set of adjustable diffusers, some made of tungsten and some of brass. The cooling cell was composed of a liquid hydrogen or lithium hydride absorber placed inside a focus coil (FC) module, sandwiched between two scintillating-fibre trackers (TKU, TKD) placed in superconducting solenoids (SSU, SSD). Together, SSU, FC, and SSD formed the magnetic channel. The MICE coordinate system is such that the $z$-axis is coincident with the beam direction, the $y$-axis points vertically upwards, and the $x$-axis completes a right-handed coordinate system.

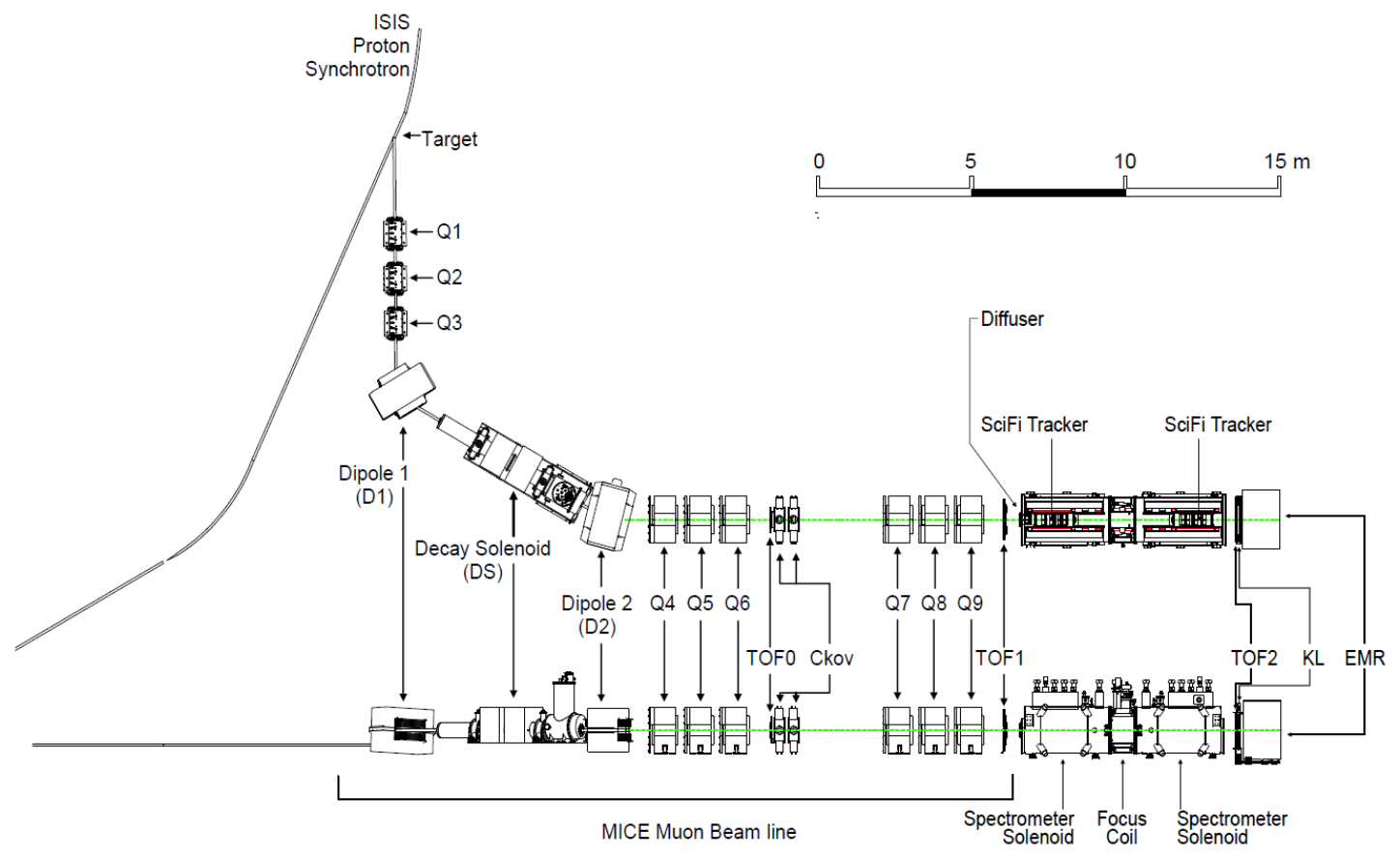

Figure 1. MICE, top (a) and side (b) views, showing the full beam line starting from the target position on the proton synchrotron with the quadrupoles and dipoles (Q1 to Q9, D1, D2), the Decay Solenoid, and instrumented magnetic channel elements (including the trackers upstream, TKU, and downstream, TKD, of the cooling channel, placed inside superconducting solenoids, respectively SSU and SSD) with all the other PID detectors (three TOF stations, two Ckov detectors, KL and the EMR). The cooling cell, defined to be the liquid hydrogen absorber vessel inside the focus coil (FC), is shown in figure 17.

MICE measured the passage of single particles through the apparatus which were aggregated into a beam offline. This paper documents the performance, during 2015-2017, of the instrumentation which was used to fully characterise the beam and its evolution along the magnetic channel, and quantifies the physical properties of the liquid hydrogen absorber. The beam instrumentation consisted of three time-of-flight detectors (TOF0, TOF1, TOF2) discussed in section 2, two threshold Cherenkov counters (CkovA, CkovB) discussed in section 3, a sampling calorimeter (KL) discussed in section 4, a tracking calorimeter (EMR) discussed in section 5, and the scintillating-fibre trackers discussed in section 6 . The properties of the liquid hydrogen absorber are described in section 7. 


\section{Time-of-flight detectors}

Three scintillator hodoscopes were used: to measure the time of flight (TOF) of the particles that made up the beam; to measure the transverse position at which the particle crossed each of the detectors; and to provide the trigger for the experiment. TOF0 and TOF1 [16-18] were placed upstream of the magnetic channel, while TOF2 [19] was located downstream of the channel, mounted in front of the KL pre-shower detector (see figure 1). At $240 \mathrm{MeV} / c$, the difference in the TOF for a muon and a pion between TOF0 and TOF1 was about $1.3 \mathrm{~ns}$. The system was therefore designed to measure the TOF with a precision of $100 \mathrm{ps}$. This allowed the TOF between the first pair of TOF stations to be used to discriminate between pions, muons, and electrons, contained within the beam, with near $100 \%$ efficiency [20]. In addition, by assuming a mass hypothesis for each particle, the TOF measurement was used to infer the particle momentum. The TOF detectors, which operated smoothly during the running periods, were essential for all the measurements that were performed [15, 20-24].

Each TOF station was made of two planes of 1 inch thick scintillator bars oriented along the $x$ and $y$ directions. The bars of TOF0 (TOF1, TOF2) were made of Bricon BC-404 (BC-420) plastic scintillators. A simple fishtail light-guide was used to attach each end of each bar to Hamamatsu R4998 fast photomultiplier tubes (PMTs). Each PMT was enclosed in an assembly that included the voltage divider chain and a $1 \mathrm{~mm}$ thick $\mu$-metal shield. For TOF1 and TOF2 an additional soft iron (ARMCO) local shield was also used $[25,26]$. The shield was required to reduce the stray magnetic field within the PMT to a negligible level [18]. To increase the count-rate stability, active dividers were used. One TOF detector is illustrated in figure 2.
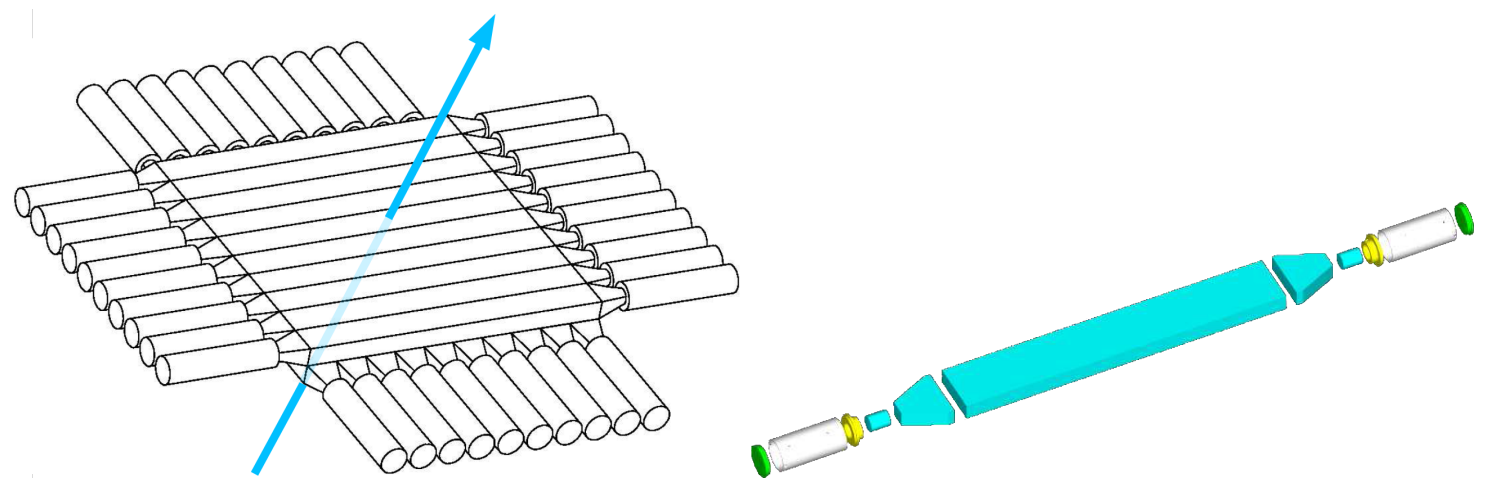

Figure 2. The structure of the time-of-flight detectors $[16,18]$ showing the horizontal and vertical layers of slabs (left) and an exploded view of each slab (right). The components of each slab are the central scintillator bar, two fishtail, clear plastic light-guides coupled to clear plastic matching pieces, and two PMTs. The beam direction is represented by the blue arrow perpendicular to the slabs.

The active areas of the three hodoscopes were $40 \times 40 \mathrm{~cm}^{2}$ (TOF0), $42 \times 42 \mathrm{~cm}^{2}$ (TOF1), and $60 \times 60 \mathrm{~cm}^{2}$ (TOF2). Each of the planes in TOF0 and TOF2 had 10 slabs while those in TOF1 had 7. A passive splitter was used to take the signal from each of the PMTs to a LeCroy 4115 leading-edge discriminator followed by a CAEN V1290 TDC for time measurement and to a CAEN V1724 FADC for pulse-height measurement. A local readout trigger was issued if the signals from each of the two PMTs on a single slab crossed a specific threshold and overlapped. TOF1 was used to trigger the readout of the experiment for most of the data taking. 
Calibration. The intensity of the scintillation light produced when a particle crossed the plastic scintillator rose rapidly before decaying with a characteristic time of $1.8 \mathrm{~ns}$. The scintillation light travelled from the particle-crossing point to each end of the scintillator slab. The light's travel time depended on the distance of the particle crossing from the PMT. The propagation speed of the light pulse along the slabs was determined to be $13.5 \mathrm{~cm} / \mathrm{ns}$.

The local readout-trigger signal was distributed to all TDC boards and was used as the reference time. The time between a particle hit in a TOF slab and the time when the trigger was generated varied with the position of the hit along the slab. As a consequence, the reference time had an offset dependent on the crossing position, an effect referred to as the readout-trigger signal delay. To compensate for this, the final time measurement in each station was an average of the times recorded for each channel above threshold.

Further delay was introduced by the signal-transit time of each PMT and of the cable that led the signal to the readout electronics. These signal-transit times were unique for each individual readout channel and were determined by dedicated measurements. The use of a linear, leading-edge discriminator led to a correlation between the total charge in the pulse and the time at which the discriminator fired. This correlation, referred to as the time-walk, introduced a systematic offset in the time recorded by the TDC that was dependent on the pulse height.

Precise determination of the TOF required a calibration procedure that allowed channel-bychannel variations in the response of the system to be accounted for. The calibration procedure described in [27] accounted for each of the effects identified above.

Reconstruction. A particle crossing a TOF station passed through two orthogonal slabs. Signals from each PMT were corrected for time-walk, readout-trigger signal delay, and the channel-specific delays. The slab-crossing time was taken to be the average of the corrected PMT times. Two slab signals were taken to have been produced by the passage of a particle if their slab-crossing times were within a $4 \mathrm{~ns}$ window. These two matched slabs were used to define a pixel of area given by the width of the slabs. The particle-crossing time was then determined as the average of the slab-crossing times and the approximate position of the particle crossing was refined using the PMT signals in the two orthogonal slabs.

Performance. The difference, $\Delta t$, between the slab-crossing times for matched slabs was used to determine the intrinsic time resolution, $\sigma_{t}$ of the TOF system. The $\Delta t$ resolution, $\sigma_{\Delta t}$, is given by $\sigma_{\Delta t}=2 \sigma_{t}$, assuming that the intrinsic resolution is the same in each of the planes that make up a particular TOF station. Figure 3 shows the distributions of $\Delta t$ for TOF0, TOF1, and TOF2 for a representative set of data taken in 2017. The RMS width of the distributions are $114 \mathrm{ps,} 126 \mathrm{ps}$, and 108 ps for TOF0, TOF1, and TOF2 respectively. The distributions are similar, and the RMS of each distribution is consistent with the measured intrinsic resolution of approximately $60 \mathrm{ps}$ [18].

Figure 4 shows an example distribution of the measured TOF between TOF0 and TOF1. The TOF peaks characteristic of electrons, muons, and pions are clearly separated. The width of the electron peak is approximately $0.10 \mathrm{~ns}$, consistent with the spread calculated from a naive quadrature addition of the timing resolution of the individual TOF stations. 

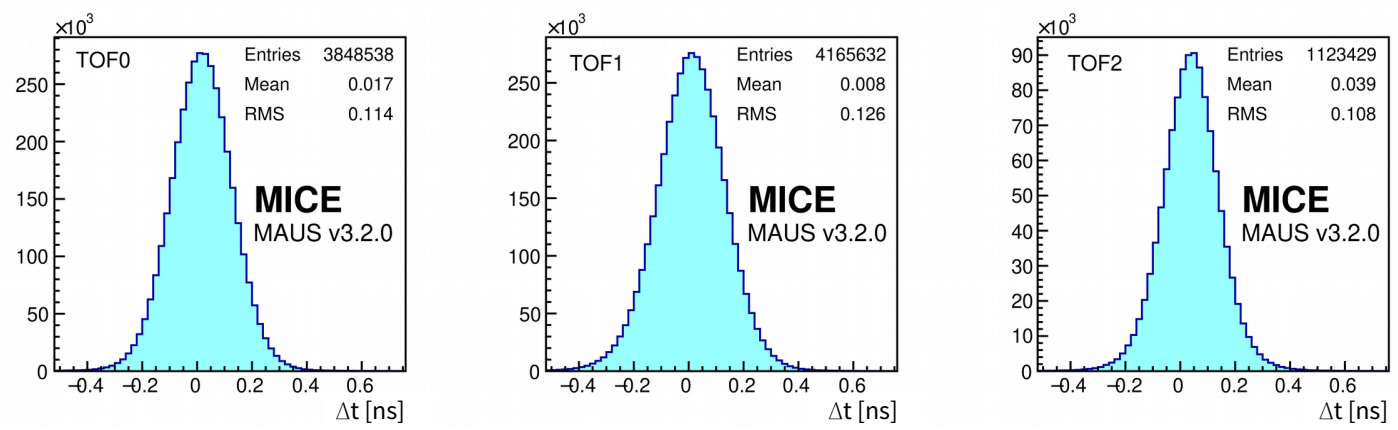

Figure 3. Slab $\Delta t$ distributions. Total width of the distribution is due to the resolution of the individual channels and due to the offsets in their $\Delta t$ distributions.

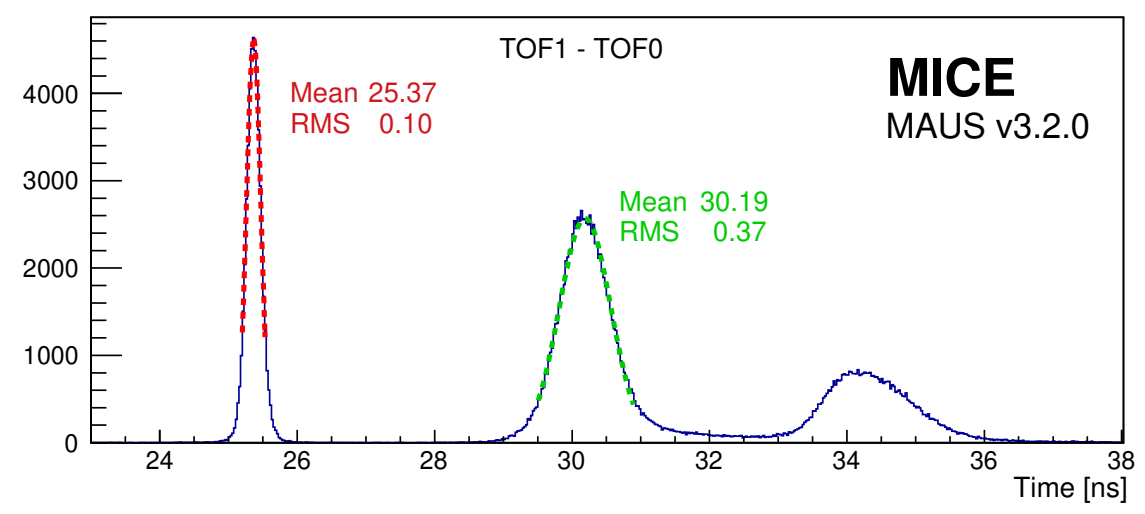

Figure 4. Time of flight between TOF0 and TOF1 after all corrections have been applied. The electron (left-most peak, shown in red), the muon (central peak, shown in green), and the pion (right-most peak, shown in blue) peaks are clearly separated.

\section{Cherenkov detectors}

The threshold Cherenkov counters were designed to distinguish muons from pions at particle momenta $\gtrsim 200 \mathrm{MeV} / c$, where the precision of the time-of-flight measurement was not sufficient for conclusive identification. Two high-density silica aerogel Cherenkov detectors with refractive indices $n=1.07$ (CkovA) and $n=1.12$ (CkovB) were used [28]. The structure of the detectors is shown in figure 5. Light was collected in each counter by four eight-inch, UV-enhanced PMTs and recorded using CAEN V1731 FADCs [29]. The two detectors were placed directly one after the other in the beamline and located just after TOF0.

The refractive indices of CkovA and CkovB result in detection thresholds for muons of approximately $280 \mathrm{MeV} / c$ and $210 \mathrm{MeV} / c$ respectively. For pions, the thresholds are approximately $367 \mathrm{MeV} / c$ (CkovA) and $276 \mathrm{MeV} / c$ (CkovB). MICE was designed to operate using beams with a central momentum between $140 \mathrm{MeV} / c$ and $240 \mathrm{MeV} / c$. The Cherenkov counters' thresholds were chosen to provide muon identification for beams of $210 \mathrm{MeV} / c$ and above, while the TOFs provide muon identification for beam below $210 \mathrm{MeV} / c$. Unambiguous identification of particle species using the Cherenkovs exploited the momentum measurement provided by the trackers. 


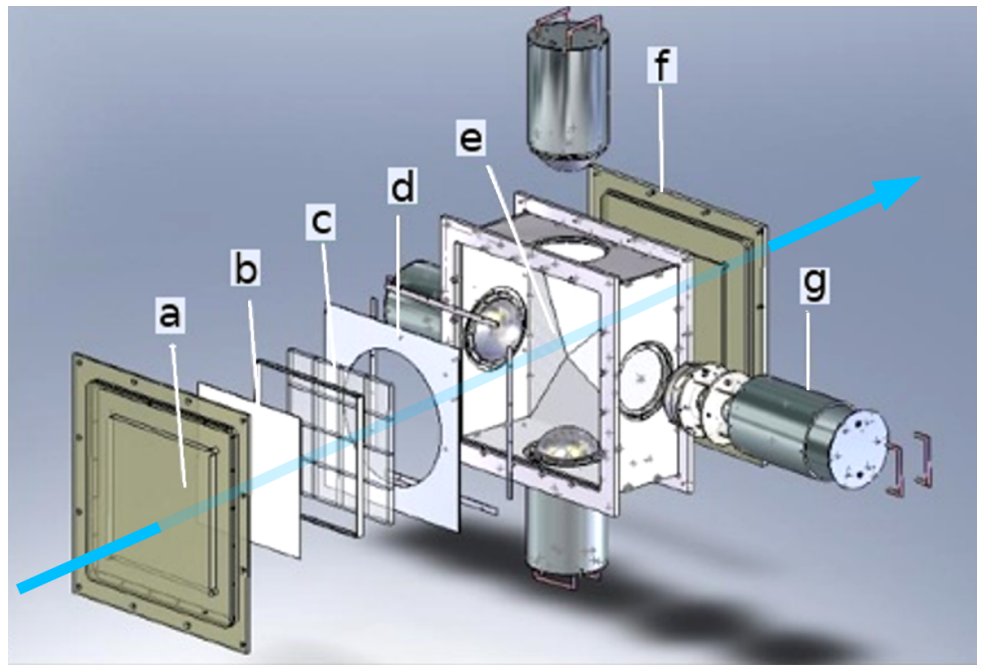

Figure 5. MICE aerogel Cherenkov counter: a) entrance window, b) mirror, c) aerogel mosaic, d) acetate window, e) GORE DRP reflector panel, f) exit window and g) eight-inch PMT in iron shield. The beam direction is represented by the blue arrow traversing the detector.

Performance. The performance of the detectors was determined using beams for which the momentum range was broad enough to observe the turn-on points and to allow the asymptotic light yields (as the particle velocity divided by the speed of light, $\beta$, approaches 1 ) to be obtained from fits to the data. The normalised photo-electron yields observed in CkovA and CkovB are plotted as a function of $\beta \gamma$ (where $\gamma=\left(1-\beta^{2}\right)^{-\frac{1}{2}}$ ) in figure 6. The pedestal in the photo-tube response arising from background photons has been subtracted. The approximate turn-on points for CkovA and CkovB were found at $\beta \gamma \approx 2.6$ and $\approx 2.1$ respectively, corresponding to refractive indices of $n \approx 1.07$ and $\approx 1.11$ which are in broad agreement with the properties of the aerogel radiators.
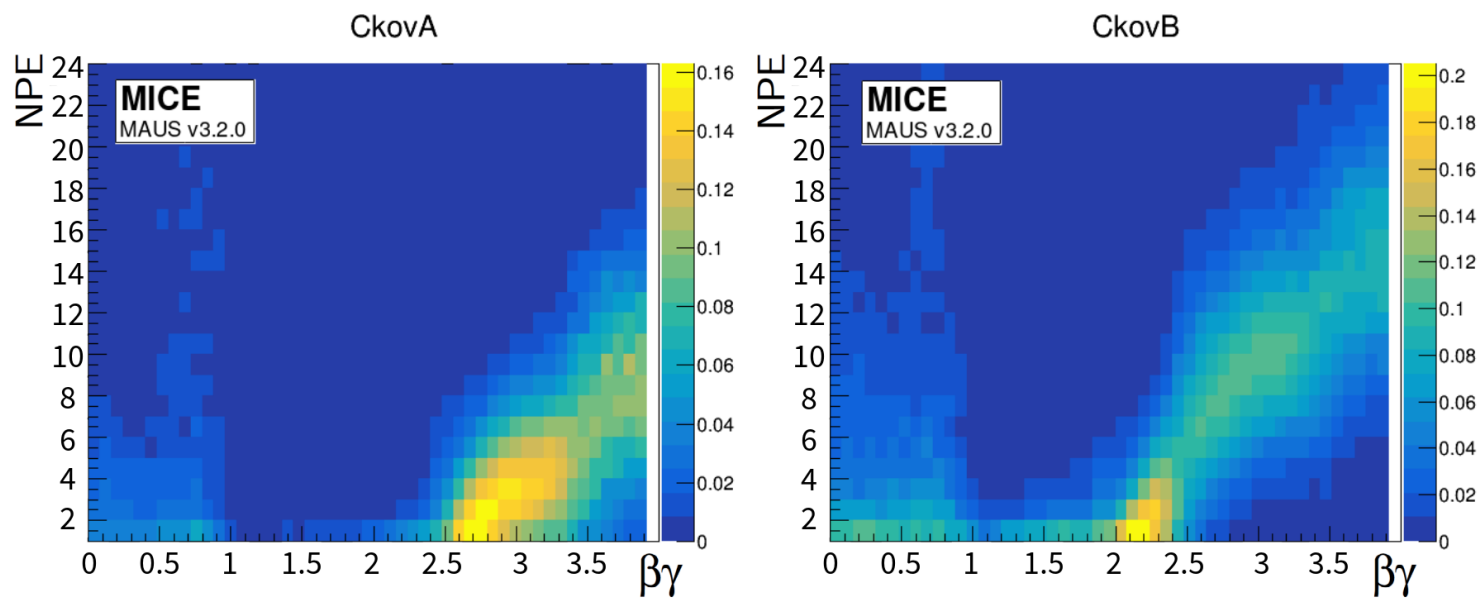

Figure 6. Photoelectron yields versus $\beta \gamma$ in CkovA and CkovB, where $\beta c$ is the particle velocity and $\gamma=\left(1-\beta^{2}\right)^{-\frac{1}{2}}$. 


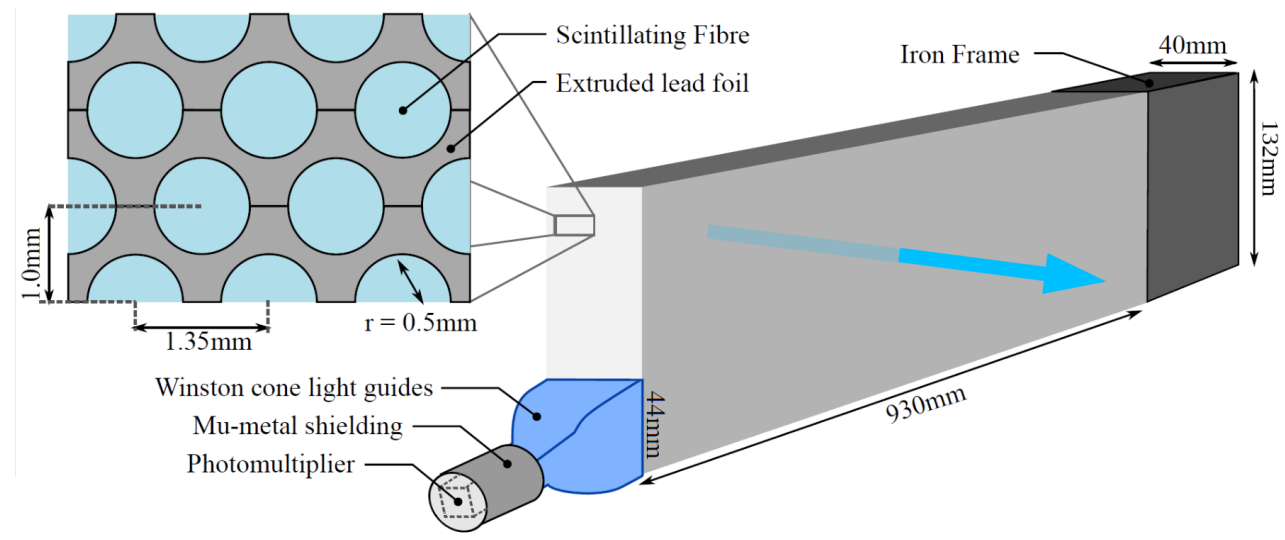

Figure 7. Single slab design of MICE KLOE-Light Calorimeter [31]; only one of the six PMT assemblies is shown. The beam direction is represented by the blue arrow traversing the slab.

\section{KLOE-light calorimeter}

The KLOE-Light (KL) pre-shower sampling calorimeter was composed of extruded lead foils in which scintillating fibres were placed. At normal incidence the thickness of the detector was 2.5 radiation lengths. The detector provided energy deposition and timing information and was used to distinguish muons from decay electrons [20]. The KL consisted of a series of layers of $1 \mathrm{~mm}$ diameter BICRON BCF-12 scintillating fibres embedded in an appropriately shaped lead sheets (see figure 7). Each fibre was separated by $1.35 \mathrm{~mm}$ from its neighbours within a layer and the distance between the centres of the fibres in adjacent layers was $0.98 \mathrm{~mm}$. One layer was shifted by half the fibre pitch with respect to the next. The volume ratio of scintillator to lead was approximately 2:1, "lighter" than the ratio of 1:1 used in the similar calorimeter of the KLOE experiment [30]. Lead/scintillator layers were stacked into slabs, $132 \mathrm{~mm}$ in depth. A total of 7 slabs formed the whole detector, which had an active volume of $93 \mathrm{~cm} \times 93 \mathrm{~cm} \times 4 \mathrm{~cm}$. Scintillation light was guided from each slab into a total of six PMTs (three at each end). Iron shields were fitted to each photomultiplier to mitigate the effect of stray magnetic fields. The signal from each PMT was sent to a shaping amplifier module that stretched the signal in time to match the sampling rate of the CAEN 1724 FADCs.

Performance. To study the response of the KL, the particle momentum was determined from the measured time-of-flight between TOF0 and TOF1. To compensate for the effect of attenuation the performance was evaluated in terms of the "ADC product" given by:

$$
\mathrm{ADC}_{\text {prod }}=\frac{2 \times \mathrm{ADC}_{\text {left }} \times \mathrm{ADC}_{\text {right }}}{\left(\mathrm{ADC}_{\text {left }}+\mathrm{ADC}_{\text {right }}\right)}
$$

where $\mathrm{ADC}_{\text {left }}$ and $\mathrm{ADC}_{\text {right }}$ are the signals from the two ends of a slab and the factor of 2 is present for normalisation. Data was taken with no field in the spectrometer solenoids or the focus coil at beam-momentum settings chosen to span the range of momenta used during MICE running. The resulting momentum distributions were centred at 140,170, 200, 240, and $300 \mathrm{MeV} / c$. The response of the KL to muons and pions was observed to increase with beam momentum. 

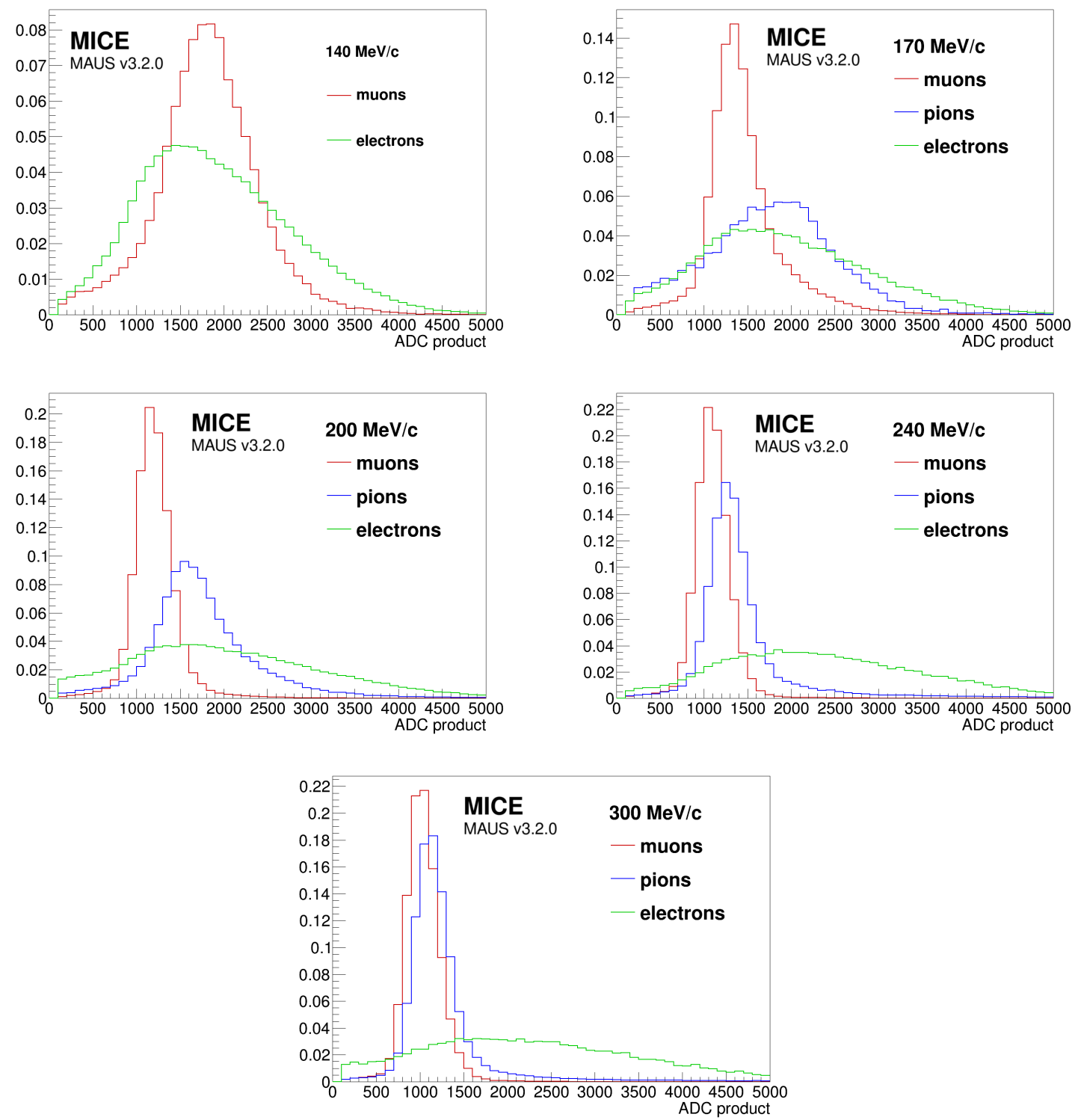

Figure 8. Comparison of ADC products of muons (red), pions (blue) and electrons (green) traversing the $\mathrm{KL}$, at $140 \mathrm{MeV} / c$ (top left), $170 \mathrm{MeV} / c$ (top right), $200 \mathrm{MeV} / c$ (middle left), $240 \mathrm{MeV} / c$ (middle right) and $300 \mathrm{MeV} / c$ (bottom).

Figure 8 presents a comparison of the response to muons, pions and electrons for various beam momentum settings. At high momentum, for example $300 \mathrm{MeV} / c$, the ADC product distributions for muons and pions are similar. At lower momentum the distributions become increasingly dissimilar, the pions having a broader distribution arising from hadronic interactions. The difference between the detector's response to pions and muons has been exploited to determine the pion contamination in the muon beams used for the MICE cooling measurements [20].

The ADC product distribution measured using a $300 \mathrm{MeV} / \mathrm{c}$ beam is compared to the MAUS [32] simulation of the detector response in figure 9. The simulation takes into account 

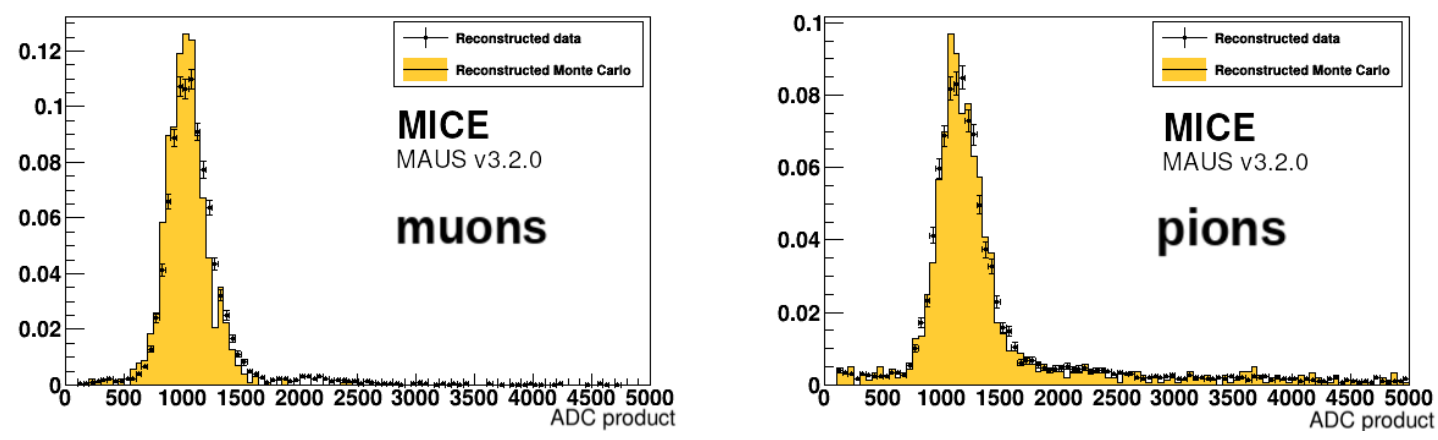

Figure 9. Comparison between data and Monte Carlo simulation of KL response to muons (left) and pions (right) at $300 \mathrm{MeV} / c$.

the light production distribution of the scintillating fibres, and the response of the PMTs for which the gain was approximately $2 \times 10^{6}$. The data is well described by the simulation.

\section{Electron muon ranger}

The EMR was a fully-active scintillator detector [33] with a granularity that allowed track reconstruction. The EMR consisted of extruded triangular scintillator bars arranged in planes. Each plane contained 59 bars and covered an area of $1.27 \mathrm{~m}^{2}$. Figure 10 shows the bar cross section and the arrangement of the bars in a plane. Triangular bars were chosen so that tracks moving parallel to the detector axis could not travel along the gaps between bars. Successive planes were mounted perpendicularly, so that hits in neighbouring planes defined a position. A single "X-Y module" was a pair of orthogonal planes. The scintillation light was collected using a wavelength shifting (WLS) fibre glued inside each bar. At each end, the WLS fibre was coupled to clear fibres that transported the light to a PMT. All the WLS fibres from one edge of a plane were read out using one single-anode PMT (SAPMT) so that an integrated charge measurement could be used to determine the energy deposited in the plane. The signals from the fibres emerging from the other edge of the plane were recorded individually using multi-anode PMTs (MAPMTs). The full detector was made up of $24 \mathrm{X}-\mathrm{Y}$ modules giving a total active volume of approximately $1 \mathrm{~m}^{3}$.

Measurements of the performance of the completed detector demonstrated an efficiency per plane of $99.73 \pm 0.02 \%[33,34]$. The level of crosstalk was within acceptable values for the type of MAPMT used, with an average of $0.20 \pm 0.03 \%$ between adjacent channels and a mean amplitude equivalent to $4.5 \pm 0.1 \%$ of the primary signal. Only four dead bars were present.

The primary purpose of the EMR was to distinguish between a muon that crossed the entire magnetic channel and those which decayed in flight producing an electron. Muons and electrons exhibited distinct behaviours in the detector. A muon produced a single straight track before either stopping or exiting the scintillating volume. Electrons showered in the lead of the KL and created a broad cascade of secondary particles. Two main geometric variables, the "plane density" and the "shower spread", were used to differentiate them. The detector was capable of identifying electrons with an efficiency of $98.6 \%$, providing a purity for the MICE beam that exceeds $99.8 \%$. 

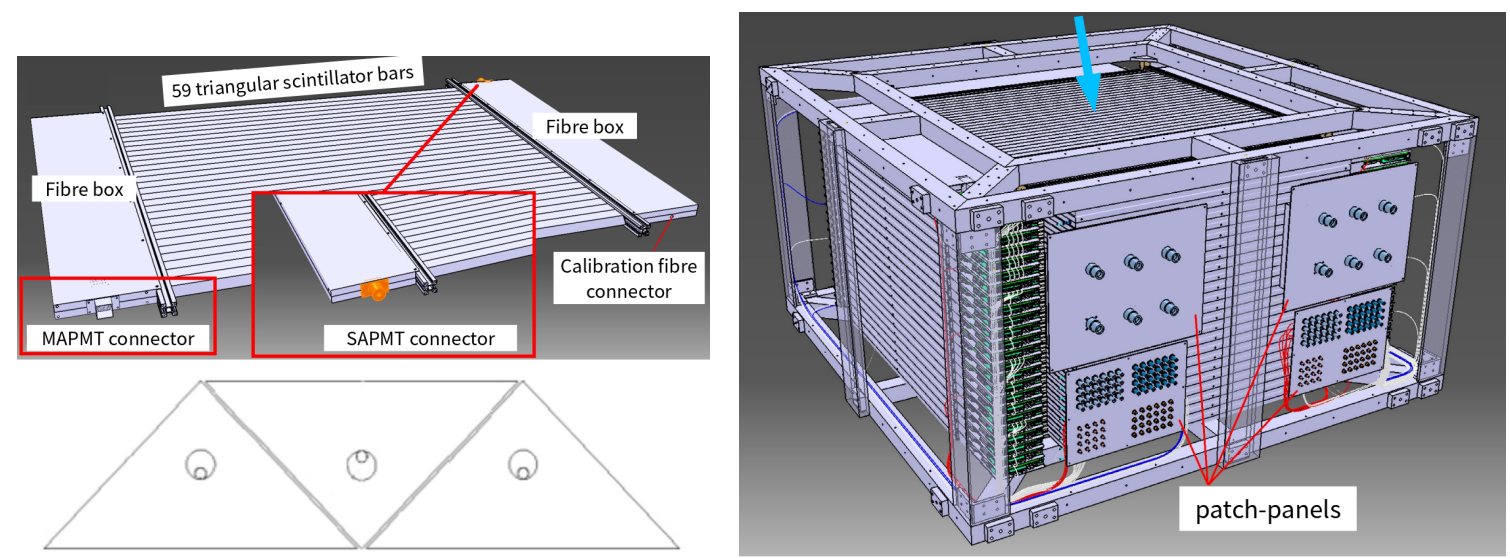

Figure 10. Drawing of one EMR plane (top left), cross section of the arrangement of 3 bars and their wavelength shifting fibres (bottom left) and drawing of the full detector and its supporting structure from a top perspective (right). The beam direction is represented by the blue arrow perpendicular to the detector.

The EMR also proved to be a powerful tool for the reconstruction of muon momenta in the range $100-280 \mathrm{MeV} / c[23]$.

Performance. A full description of the detector and the reconstruction algorithms used may be found in reference [23]. Here the performance of the EMR detector over the course of the experiment is summarised.

To measure the performance of the EMR the MICE beamline was set to deliver a nominal momentum of $400 \mathrm{MeV} / c$. This maximised the muon transmission to the EMR and its range in the detector. In this configuration the beamline produced pions and muons in comparable quantities, as well as a smaller number of electrons. Time-of-flight between TOF1 and TOF2 was used to identify particle species and only particles compatible with the muon hypothesis were included in the analysis. Particles entering the muon sample had a momentum larger than $350 \mathrm{MeV} / \mathrm{c}$ at the upstream surface of TOF2 and were expected to cross both TOF2 and the KL and penetrate the EMR. $99.62 \pm 0.03 \%$ of the particles entering TOF2 were observed to produce hits in the EMR. The small inefficiency may be attributed to pions in the muon sample that experienced hadronic interactions in the KL. If hits were produced in the detector, an $(x, y)$ pair, defining a space point, was reconstructed $98.56 \pm 0.06 \%$ of the time.

To evaluate the efficiency of the scintillator planes, only the muons that traversed the entire detector were used. Muons were selected which produced a hit in the most downstream plane. For these events a hit was expected in at least one bar in each plane on its path. The mode of the hit-multiplicity distribution per plane was one, in $3.26 \pm 0.02 \%$ of cases a plane traversed by a muon did not produce a signal in the MAPMT, and the probability that the track was not observed in the SAPMT was $1.88 \pm 0.01 \%$.

Electron rejection. A broad range of beamline momentum settings was used to characterise the electron-rejection efficiency. Particle species were characterised upstream of the EMR using the time-of-flight between TOF1 and TOF2. For each momentum setting, a fit was carried out to determine the position of the muon and electron time-of-flight peaks and events were selected 
accordingly to form muon and electron-template samples. Particles with a time-of-flight larger than the upper limit of the muon sample were either pions or slow muons and were rejected.

To distinguish the muon tracks from the electron-induced showers, two particle-identification variables were defined based on the distinct characteristics of the two particle species. The first is the plane density, $\rho_{p}$ :

$$
\rho_{p}=\frac{N_{p}}{Z_{p}+1},
$$

where $N_{p}$ is the number of planes hit and $Z_{p}$ the number of the most downstream plane [23]. A muon deposits energy in every plane it crosses until it stops, producing a plane density close to one. An electron shower contains photons that may produce hits deep inside the fiducial volume without leaving a trace on their path, reducing the plane density. The second variable is the normalised $\hat{\chi}^{2}$ of the fitted straight track given by

$$
\hat{\chi}^{2}=\frac{1}{N-4} \sum_{i=1}^{N} \frac{\operatorname{res}_{x, i}^{2}+\operatorname{res}_{y, i}^{2}}{\sigma_{x}^{2}+\sigma_{y}^{2}} ;
$$

where $N$ is the number of space points (one per bar hit), $\operatorname{res}_{q, i}$ the residual of the space point with respect to the track in the $q z$ projection and $\sigma_{q}$ the uncertainty on the space point in the $q z$ projection, $q=x, y[35]$. This quantity represents the transverse spread of the hits produced by the particle in the EMR. A muon produced a single track giving $\hat{\chi}^{2}$ close to one, while an electron shower produced a larger value. The two discriminating variables can be combined to form a statistical test on the particle hypothesis. Dense and narrow events will be tagged as muons while non-continuous and wide showers will not. The quality of this statistical test was characterised in terms of the fraction of the muon sample that is rejected, $\alpha$, and the fraction of the electron sample that is selected, $\beta$.

The momentum of the particles was measured by the downstream tracker and this information used to determine the momentum dependence of the contamination and loss in the range 100$300 \mathrm{MeV} / c$. Figure 11 shows the loss, $\alpha$, and the contamination, $\beta$, as a function of the momentum measured in TKD. $\alpha$ increases towards low muon momentum. This is due both to an increase in the decay probability between TOF2 and the EMR and a decrease in the number of muons that cross the KL to reach the EMR.

\section{Tracking}

The MICE instrumentation allowed individual particles to be tracked from TOF0 to the EMR, a distance of more than $15 \mathrm{~m}$. High-resolution particle tracking was provided by two scintillatingfibre trackers (section 6.1). The precise relative alignment of the time-of-flight hodoscopes and the trackers was obtained by combining the measurements of both detector systems (section 6.2).

\subsection{Trackers}

The two high-precision scintillating-fibre trackers each had a sensitive volume that was $110 \mathrm{~cm}$ in length and $30 \mathrm{~cm}$ in diameter [36]. Each tracker was composed of five stations (labelled 1 to 5, with station 1 being closest to the cooling cell) held in position using a carbon-fibre spaceframe. Adjacent stations were separated by different distances ranging from $20 \mathrm{~cm}$ to $35 \mathrm{~cm}$. The 


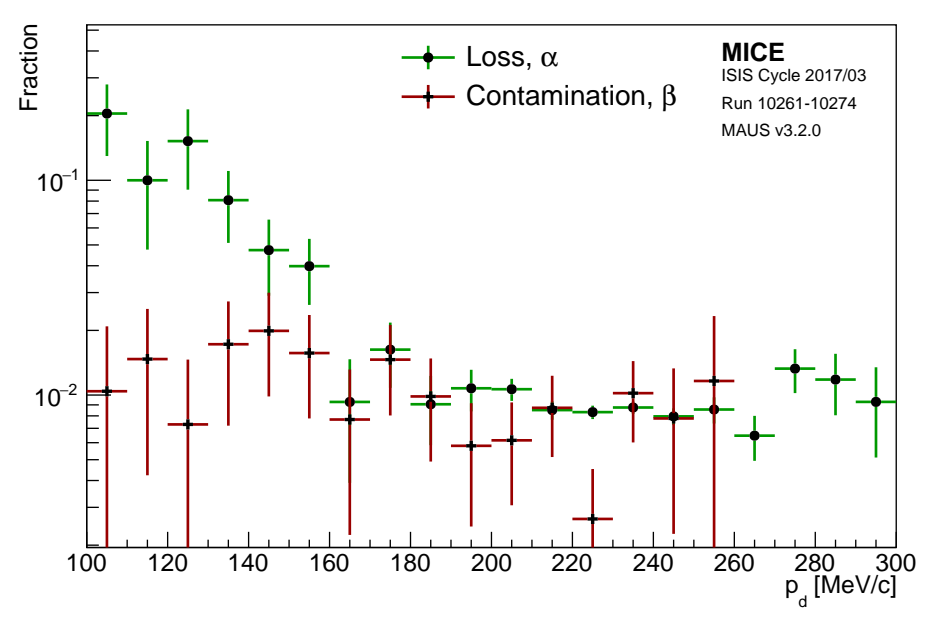

Figure 11. Percentage of electron contamination, $\beta$, and muon loss, $\alpha$, for different ranges of momentum measured in the downstream tracker, $p_{d}$. The error bars are based on the statistical uncertainty in a bin, and the bin width set by the resolution of the measurement.

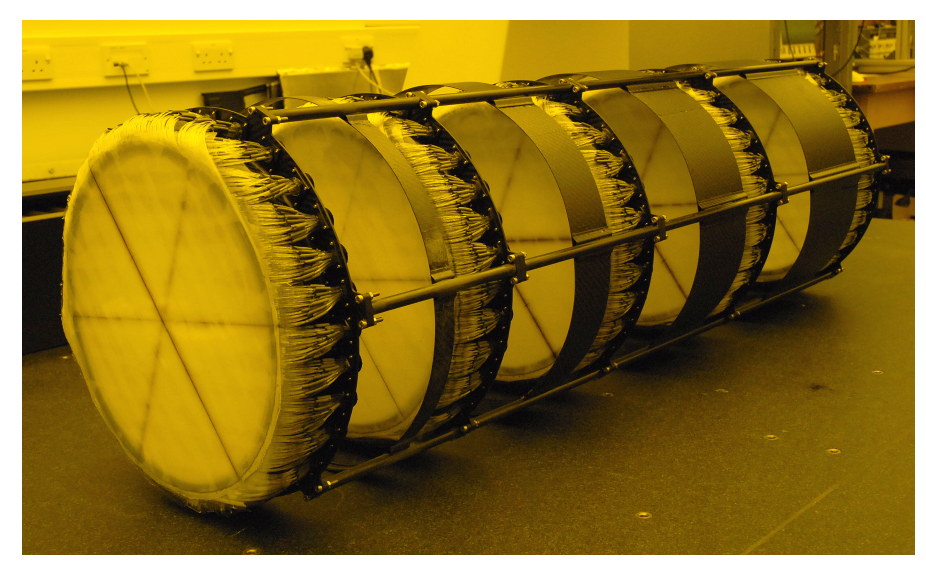

Figure 12. Photograph, with UV-filtered light, of one of the MICE trackers, showing the five stations. Each station has three doublet planes of scintillating fibres, each plane at $120^{\circ}$ to the next (the central fibres of each plane can be seen as darker lines traversing the station).

separations were chosen to ensure that the azimuthal rotation of track position did not repeat from one station to the next. This property was exploited in the ambiguity-resolution phase of the pattern recognition. Each tracker was instrumented with an internal LED calibration system and four 3-axis Hall probes to monitor the field. A photograph of one of the trackers on the bed of the coordinate measuring machine used to verify the mechanical alignment of the stations is shown in figure 12 .

Each tracker station consisted of three doublet layers of $350 \mu \mathrm{m}$ scintillating fibres; these layers were arranged such that each was set at an angle of $120^{\circ}$ with respect to the next. This arrangement ensured that there were no inactive regions between adjacent fibres. Fibres were grouped into one bundle of seven for each readout channel, to match the resolution to that imposed by multiple scattering and reduce the overall number of readout channels. This resulted in a spatial resolution per doublet layer of $470 \mu \mathrm{m}$ and a measured light yield of approximately 10 photo-electrons [36]. 
The light from the seven scintillating fibres was coupled into a single clear fibre which took it to a visible light photon counter (VLPC) [37]. The signals from the VLPCs were digitised using electronics developed by the D0 collaboration [38].

Reconstruction. The reconstruction software for the trackers is described in [39]. Each of the 15 doublet layers provided 214 readout channels. Calibration data taken without beam was used to determine the pedestal and the gain of each channel. These calibrations were used to correct the number of photoelectrons (NPE) corresponding to the signal recorded by the tracker electronics. The first step in the reconstruction was to record the unique channel number associated with each NPE value in a "digit". Digit profiles were used to identify hot or dead channels which were masked from the reconstruction to reduce the rate of ambiguities that had to be resolved in the pattern recognition and to ensure the accuracy of the calibration. The reconstruction proceeded to create "spacepoints" from the intersection of digits in adjacent doublet layers. Spacepoints were constructed from clusters from all three planes (a triplet spacepoint) or from any two out of the three planes (a doublet spacepoint). The pattern-recognition algorithm searched for spacepoints from neighbouring stations that were consistent with the helical trajectory of a charged particle in the solenoidal field. In the final stage of the tracker reconstruction the track parameters were estimated using a Kalman filter.

Noise. Digits above a certain NPE threshold were admitted to the spacepoint-finding algorithm. Noise in the electronics arising from, for example, the thermal emission of electrons, could give rise to digits passing the threshold. Any digit not caused by the passage of a charged particle was classified as noise. To isolate noise from signal during beam-on data collection, events containing a track which included a spacepoint in each of the five tracker stations were selected. All digits corresponding to the track were removed from the total set of digits and the remainder were considered to be noise. The average noise rate per channel per event was then calculated as the total number of digits above the NPE threshold divided by the number of active channels and the number of events in the sample. The result of this calculation was that, for an NPE threshold of 2 , the fraction of digits arising from noise was $0.18 \%$ in the upstream tracker and $0.06 \%$ in the downstream tracker.

Track-finding efficiency. The track-finding efficiency was determined using a sample of events for which the time-of-flight determined from hits in TOF1 and TOF2 was consistent with passage of a muon. This requirement ensured that the particle had been transmitted successfully through the magnetic channel, crossing both trackers. The track-finding efficiency was defined to be the number of events in which a track was successfully reconstructed divided by the total number of events in the sample. The results of the efficiency analysis are tabulated in table 1 for a range of nominal beam momentum and emittance settings. The track-finding efficiency obtained in this way averaged over beam conditions was $98.70 \%$ for the upstream tracker and $98.93 \%$ for the downstream tracker. The spacepoint-finding efficiency, defined as the number of spacepoints found divided by the number of space points expected, was also determined. The spacepoint-finding efficiency is summarised for a range of beam conditions in table 2 .

The efficiency of the trackers over the data taking period was evaluated by selecting events with a measured time-of-flight between TOF1 and TOF2 consistent with the passage of a muon. Events were required to contain at least one hit within the fiducial volume of the tracker. An event was added to the numerator of the efficiency calculation if it contained a single space point in each of 
Table 1. The track finding efficiency for the upstream and downstream trackers for $140 \mathrm{MeV} / c$ and $200 \mathrm{MeV} / c$ beams, and for 3, 6 and $10 \mathrm{~mm}$ nominal emittances.

\begin{tabular}{|c|c|c|c|}
\hline Momentum & Emittance & Upstream tracks found & Downstream tracks found \\
\hline $200 \mathrm{MeV} / c$ & $3 \mathrm{~mm}$ & $98.38 \%$ & $99.19 \%$ \\
$200 \mathrm{MeV} / c$ & $6 \mathrm{~mm}$ & $99.42 \%$ & $96.07 \%$ \\
$140 \mathrm{MeV} / c$ & $6 \mathrm{~mm}$ & $98.37 \%$ & $99.16 \%$ \\
$140 \mathrm{MeV} / c$ & $10 \mathrm{~mm}$ & $98.47 \%$ & $98.93 \%$ \\
\hline \multicolumn{2}{|c|}{ Average } & $98.70 \%$ & $98.21 \%$ \\
\hline
\end{tabular}

Table 2. The spacepoint-finding efficiency, in the presence of a track, for the upstream and downstream trackers for $140 \mathrm{MeV} / c$ and $200 \mathrm{MeV} / \mathrm{c}$ beams, and for 3, 6 and $10 \mathrm{~mm}$ nominal emittances.

\begin{tabular}{|c|c|c|c|}
\hline Momentum & Emittance & Upstream spacepoints found & Downstream spacepoints found \\
\hline $200 \mathrm{MeV} / c$ & $3 \mathrm{~mm}$ & $98.04 \%$ & $97.41 \%$ \\
$200 \mathrm{MeV} / c$ & $6 \mathrm{~mm}$ & $99.41 \%$ & $94.63 \%$ \\
$140 \mathrm{MeV} / c$ & $6 \mathrm{~mm}$ & $97.99 \%$ & $99.16 \%$ \\
$140 \mathrm{MeV} / c$ & $10 \mathrm{~mm}$ & $98.07 \%$ & $97.44 \%$ \\
\hline \multicolumn{2}{|c|}{ Average } & $98.44 \%$ & $97.01 \%$ \\
\hline
\end{tabular}

the five tracker stations. The evolution of the tracking efficiency in the upstream and downstream trackers is shown in figure 13. The efficiency is shown separately for data taken in the presence of a magnetic field ("helical") and with the solenoids turned off ("straight"). The data shows that the efficiency was generally greater than $99.0 \%$. Water vapour ingress to the cold end of the VLPC cassettes caused the loss of channels and contributed to a reduction in the tracking efficiency. This was recovered by warming and drying the VLPCs.

Track-fit performance. Monte Carlo simulation with realistic field, beam conditions and detector geometry was used to estimate the performance of the track fit. A beam centred at $140 \mathrm{MeV} / c$ with $10 \mathrm{~mm}$ nominal emittance, representing a typical data set, was used for the study. Results are presented in figure 14 for the upstream tracker and figure 15 for the downstream tracker. The resolution in the total momentum and transverse momentum is observed to be $\sim 1.1 \mathrm{MeV} / c$ independent of momentum in the range $120 \mathrm{MeV} / c$ to $160 \mathrm{MeV} / c$. The small bias in the transverse and the total momentum did not give rise to significant effects in the analysis and was considered in systematic error studies.

\subsection{Beam-based detector alignment}

A beam-based alignment algorithm was developed to improve the resolution on the position of the scintillating-fibre trackers relative to the time-of-flight hodoscopes. The starting point for the beam-based alignment was the geometrical survey of the detectors in the MICE Hall which was performed using laser geodesy. Survey monuments on the TOF frames were surveyed with respect 

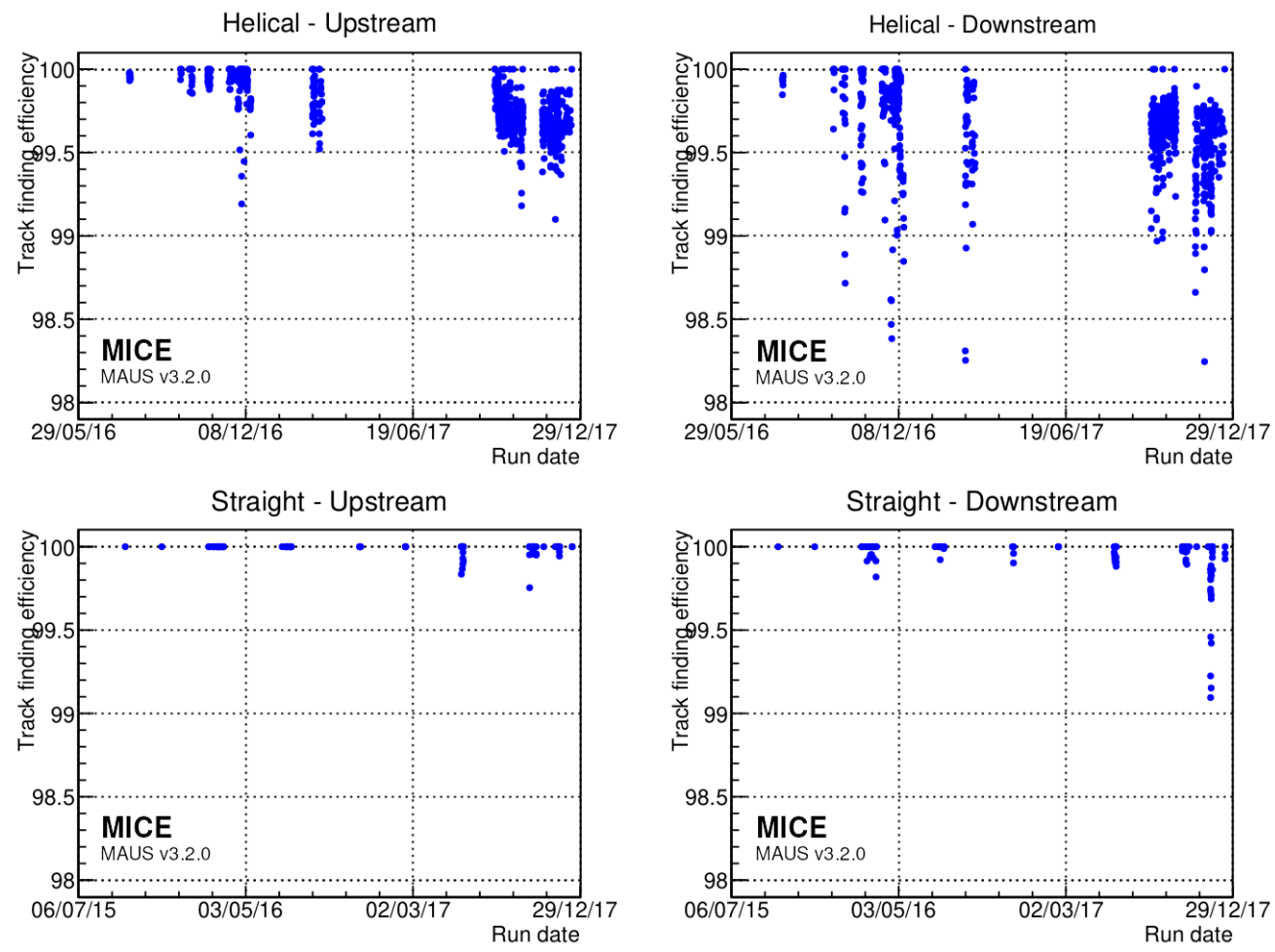

Figure 13. Evolution of the straight and helical track finding efficiencies over time for: the upstream (left); and downstream (right) trackers during the key periods of data taking since 2015. Each dot represents a single data taking run between 10 minutes and 3 hours long.

to the MICE Hall survey network. The trackers had been dowelled in position in the bores of the spectrometer solenoids. The dowels were used to locate each tracker precisely with respect to the axis of the warm bore of its solenoid. The position of the trackers along the beam line was inferred from the measurement of survey monuments mounted on the spectrometer-solenoid cryostats outer jackets. The beam-based alignment was used to determine the azimuthal orientation of the trackers with a resolution of $6 \mathrm{mrad} / \sqrt{N}$ and their position transverse to the beamline with a resolution of $20 \mathrm{~mm} / \sqrt{N}$, where $N$ is the number of tracks used in the analysis [40].

Analysis method. The position of each tracker in the MICE Hall coordinate system was described using the location of its centre and a set of three angles corresponding to rotation about the $x$ axis $(\alpha)$, the $y$ axis $(\beta)$ and the $z$ axis $(\phi)$. The rotation of the tracker about the $z$ axis has a negligible effect on the alignment since $\phi$ was determined precisely at installation. An initial estimate for the position of each tracker along the beamline had been inferred from the survey. The surveyed location of the TOFs was used as the reference for the tracker alignment. The line that joins the centre of TOF1 with the centre of TOF2 was chosen as the reference axis. A deviation from this axis was considered to be due to misalignment of the trackers. The alignment could not be determined on a single-particle basis due to multiple Coulomb scattering in the absorber and other material present on the beamline. Therefore, the mean residuals in position $(x$ and $y)$ and angle $(\alpha$ and $\beta$ ) of the trackers with respect to the TOF1-TOF2 axis were evaluated to determine the alignment constants. 

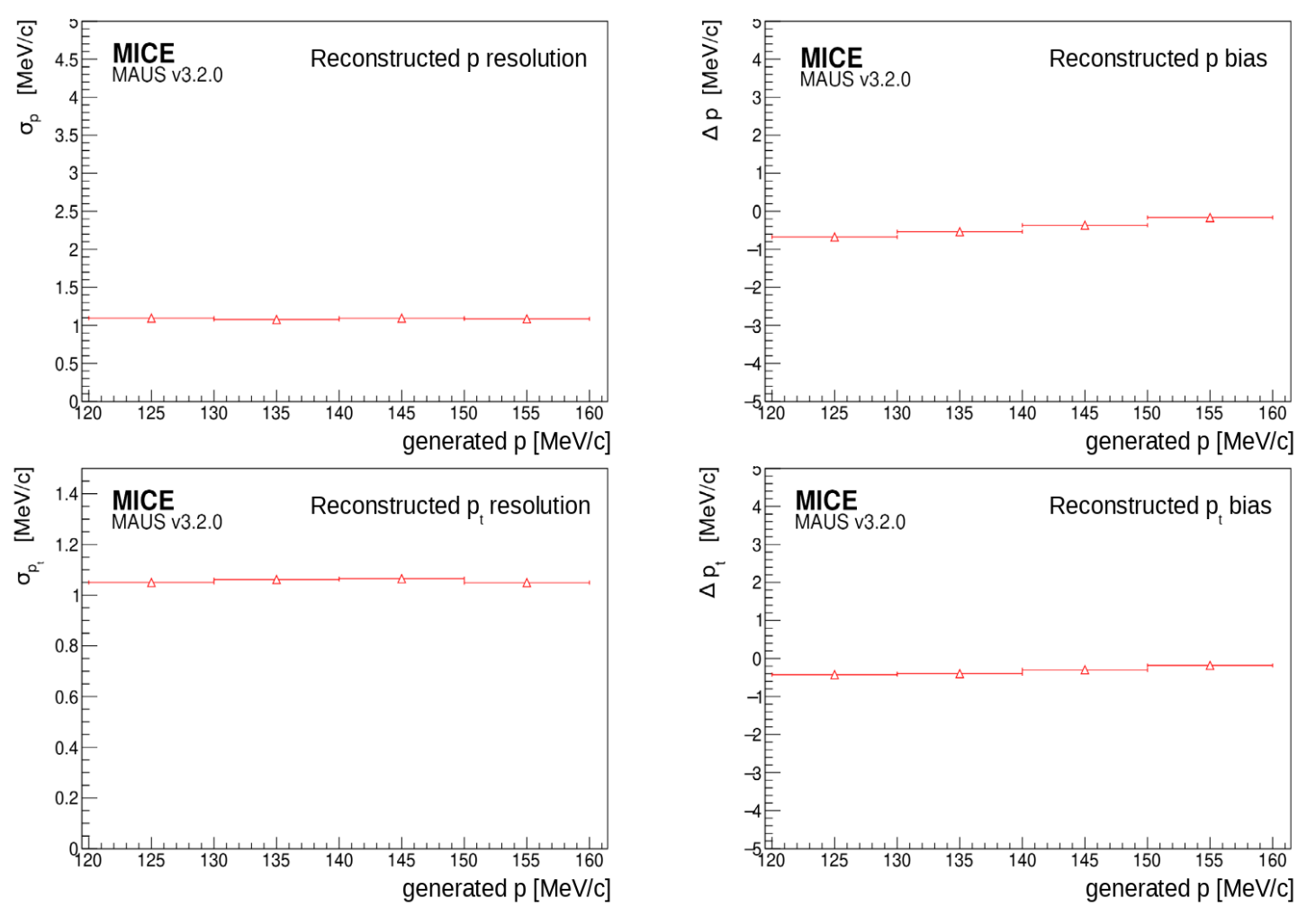

Figure 14. Momentum reconstruction resolution (left) and bias (right) for the total momentum (top) and transverse momentum component (bottom) in the upstream tracker.

Each TOF provided a single spacepoint in the Hall coordinate system. In Hall coordinates, on average, the track reconstructed between TOF1 and TOF2 should agree with the track reconstructed in each tracker, i.e. the mean residuals in $x, y, \alpha$, and $\beta$ should be zero. Applying this reasoning to the unknown offset and angles leads to a system of equations for the four unknown constants [40]. The measurement of four residual distributions per tracker yields the alignment constants. The main source of bias was the scattering in the material between TOF1 and TOF2. If the beam was not perfectly centred, particles preferentially scraped out on one side of the magnet bore, anisotropically truncating the tail of the residual distribution. A fiducial cut was applied to the upstream sample in order to remove this effect.

Data were recorded with the superconducting magnets turned off. High momentum beams were used to reduce the RMS scattering angle and to maximise transmission. Each data set was processed independently. Figure 16 shows the alignment parameters determined for each run during a specific data taking period. The measurements are in good agreement with one another and show no significant discrepancy: an agreement between the independent fits guaranteed an unbiased measurement of the alignment constants. The constant-fit $\chi^{2} /$ ndf was close to unity for each fit, indicating that there were no additional sources of significant uncertainty. The optimal parameters are summarised in table 3 . 

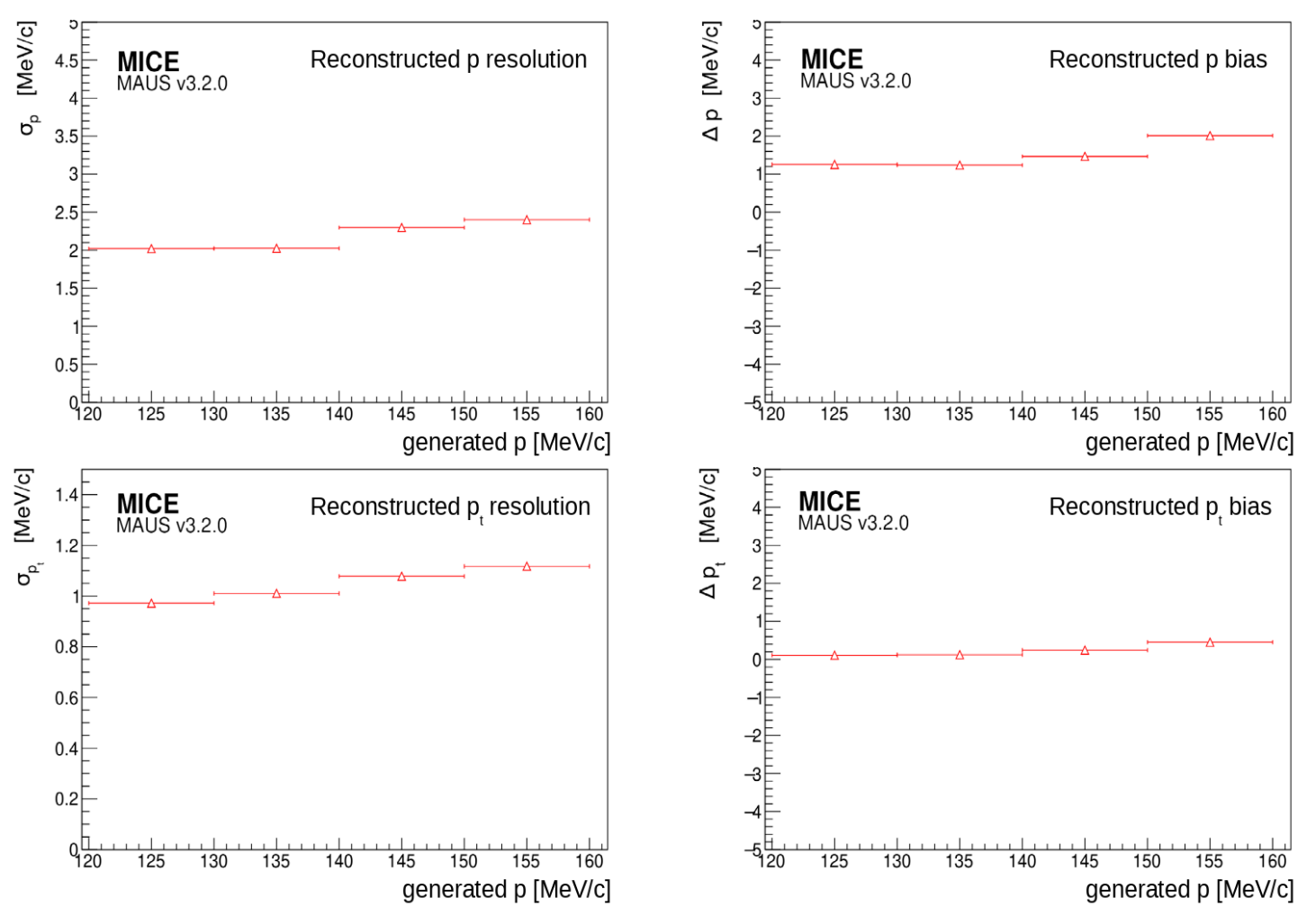

Figure 15. Momentum reconstruction resolution (left) and bias (right) for the total momentum (top) and transverse momentum component (bottom) in the downstream tracker.

Table 3. Optimal alignment constants measured in the high-momentum straight-track data acquired during May 2017 (summarised from figure 16).

\begin{tabular}{|c|c|c|c|c|}
\hline & $\mathbf{x}[\mathbf{m m}]$ & $\mathbf{y}[\mathbf{m m}]$ & $\alpha$ [mrad] & $\beta$ [mrad] \\
\hline TKU & $-0.032 \pm 0.094$ & $-1.538 \pm 0.095$ & $3.382 \pm 0.030$ & $0.412 \pm 0.029$ \\
TKD & $-2.958 \pm 0.095$ & $2.921 \pm 0.096$ & $-0.036 \pm 0.030$ & $1.333 \pm 0.030$ \\
\hline
\end{tabular}

\section{Liquid hydrogen absorber}

The accurate characterisation of the properties of the liquid hydrogen absorber was a criticallyimportant contribution to the study of ionisation cooling. The instrumentation used for this purpose and its performance are presented in this section.

The absorber vessel consisted of a cylindrical aluminium body sealed with two thin aluminium end windows, as shown in figure 17. The absorber vessel contained 221 of liquid. The body of the absorber had an inner diameter of $300 \mathrm{~mm}$ and the end flanges were separated by a distance of $230 \mathrm{~mm}$. The vessel was surrounded by a second pair of safety windows. The length along the central axis, between the two domes of the end windows, was $350 \mathrm{~mm}$ [41].

Variation of the density of liquid hydrogen due to varying temperature and pressure. The energy lost by a muon travelling through the liquid hydrogen absorber depends on the path length and 

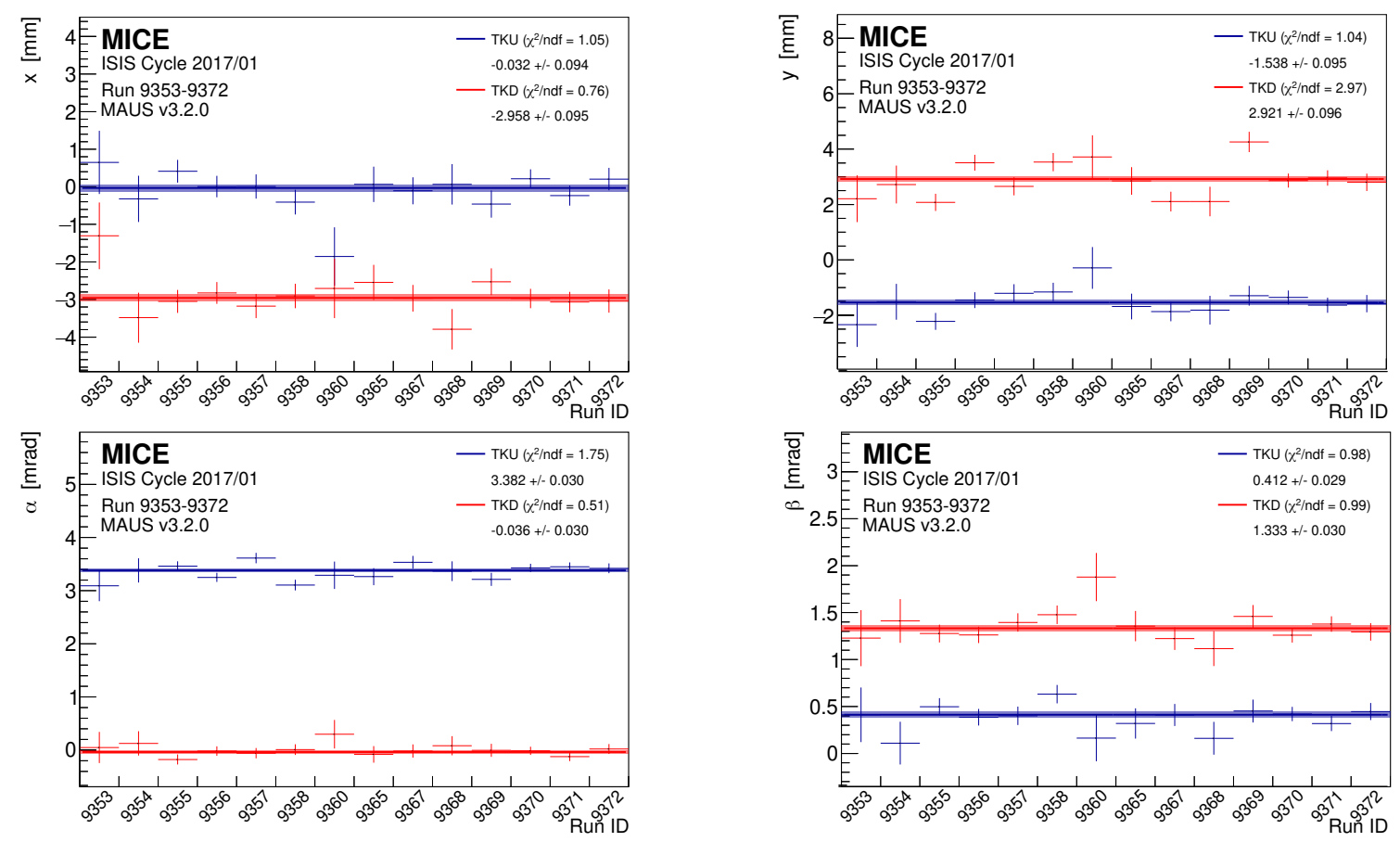

Figure 16. Consistency of the alignment algorithm results for upstream (blue) and downstream (red) trackers across runs acquired during the 2017/01 ISIS user cycle. The quantities $x, y, \alpha$, and $\beta$ are defined in the text.
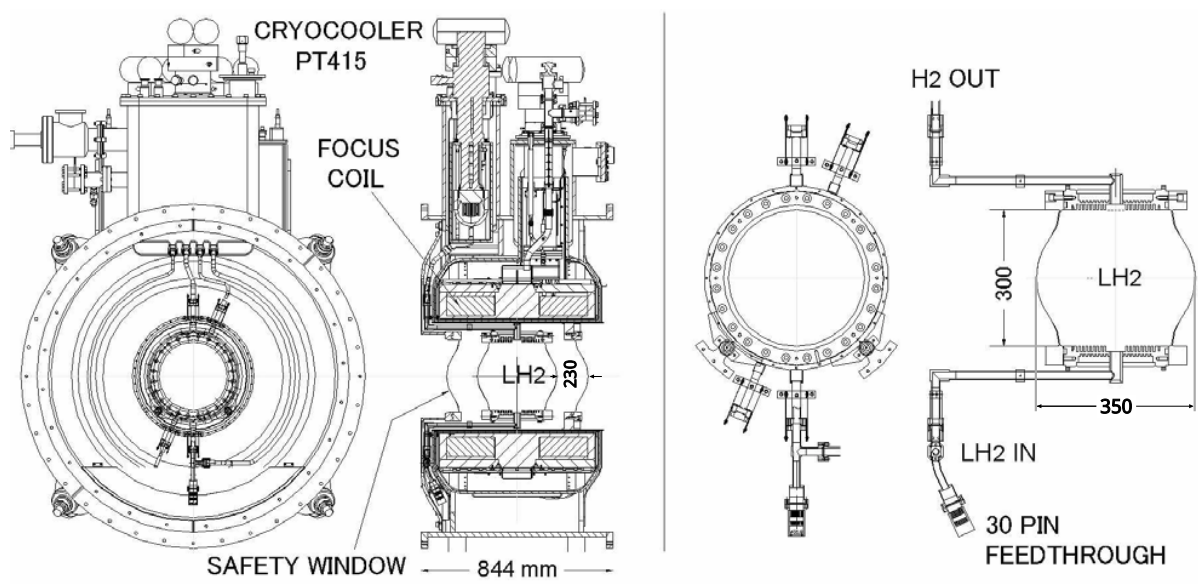

Figure 17. Left panel: drawing of the focus coil (FC) module showing the principal components. Right panel: detail of the liquid hydrogen absorber vessel [41].

on the density of the liquid hydrogen. The density of liquid hydrogen is a function of temperature and pressure. The temperature of the vessel was measured by eight LakeShore Cernox 1050 SD sensors, but with the values truncated for storage at a granularity of $0.1 \mathrm{~K}$. Four of the sensors were used solely as temperature sensors, while the other four were also used as level sensors to ensure the liquid hydrogen reached the top of the vessel. The sensors were arranged in pairs, with two mechanically clamped at the top of the vessel, two at a polar angle of $45^{\circ}$ to vertical from the top of the vessel, two at a polar angle of $45^{\circ}$ to the bottom of the vessel, and a final two at the bottom of the vessel. 
Cooldown and liquefaction were completed slowly over eight days at a pressure of $1105 \mathrm{mbar}$ after which the vessel's pressure was lowered to 1085 mbar [41]. The vessel then remained in this steady state during the 21 day period of data taking, after which the vessel was vented. For the venting process, the cryocooler used to liquefy hydrogen was switched off and heaters were switched on to deliver a nominal power of $50 \mathrm{~W}$ to the absorber vessel. This resulted in an increase in pressure to 1505 mbar until the temperature stabilised at the boiling point. A rapid increase in temperature was observed once all the liquid hydrogen had boiled off.

The temperature sensors had a typical accuracy of $\pm 9 \mathrm{mK}$ and a long-term stability of $\pm 12 \mathrm{mK}$ at $20 \mathrm{~K}$. The magnetic-field dependent temperature error, $\Delta T / T$, at $2.5 \mathrm{~T}$ is $0.04 \%$, equivalent to $\pm 8 \mathrm{mK}$ at $20 \mathrm{~K}$ [42]. These uncertainties were quoted by the manufacturer of the sensors. Magnetic fields caused reversible calibration shifts on the temperature measurements. To reduce the uncertainty in the liquid hydrogen density a calibration procedure was devised that used the boiling point, as observed during the venting process. A correction to the observed temperature reading was obtained by applying a cut-off correction, a correction for the effect of the magnetic field based on the current in the focus coil and its polarity, a correction for the non-linearity of the sensors, and a boiling point scaling factor [43].

The boiling point of hydrogen at $1085 \mathrm{mbar}$ is $20.511 \mathrm{~K}$. The sensors had a total uncertainty of $17 \mathrm{mK}$ ( $9 \mathrm{mK}$ accuracy, $12 \mathrm{mK}$ stability, $8 \mathrm{mK}$ magnetic). The deviation from the non-linearity of the sensors [42] added, on average, $0.03 \mathrm{~K}$ to the uncertainty. The temperature scaling and magnetcurrent correction factors also had an associated uncertainty as they were derived based on the $0.1 \mathrm{~K}$ resolution of the retrieved, truncated, values. For example, a calibrated sensor at boiling temperature and 1505 mbar should read $21.692 \mathrm{~K}$, but we can only retrieve a value of $21.65 \mathrm{~K}$ ( $21.6 \mathrm{~K}$ truncated plus $0.05 \mathrm{~K}$ cut-off correction [43]) i.e. off by $0.042 \mathrm{~K}$. The pressure sensors had an uncertainty of \pm 5 mbar which equated to $\pm 0.016 \mathrm{~K}$ during steady state. The pressure uncertainty ( \pm 5 mbar) added another uncertainty to the temperature calibration constants of $\pm 0.014 \mathrm{~K}$. Collectively, all these uncertainties summed in quadrature to $0.2 \mathrm{~K}$ for each sensor.

While in the steady state condition the liquid hydrogen was close to the boiling temperature of liquid parahydrogen [43] (density of $70.53 \mathrm{~kg} / \mathrm{m}^{3}$ ): the average temperature of the eight sensors was $(20.51 \pm 0.07) \mathrm{K}$ at $1085 \mathrm{mbar}$ (figure 18 ) allowing us to determine the uncertainty in the density over this period as $0.08 \mathrm{~kg} / \mathrm{m}^{3}$.

Contraction of the absorber vessel due to cooling. The absorber was cooled from room temperature to the operating temperature of the experiment $(20.51 \mathrm{~K})$, contracting the vessel. The linear contraction of Al-6061 as it is cooled from $293 \mathrm{~K}$ is given by:

$$
\alpha=-4.1277 \times 10^{-3} T-3.0389 \times 10^{-6} T^{2}+8.7696 \times 10^{-8} T^{3}-9.9821 \times 10^{-11} T^{4}
$$

where $T$ is the operating temperature [44]. The equation is the result of a fit to data collated by the National Institute of Standards and Technology (NIST) and has an associated curve fit error of $4 \%$. At the MICE operating temperature, this corresponds to a linear contraction of the vessel along each plane of $0.415 \%$. As a result the length of the bore contracted by $(1.45 \pm 0.05) \mathrm{mm}$. The vessel was suspended within the warm bore of the focus coil and was therefore free to contract in each plane without restriction. 
Liquid Hydrogen Temperature during steady-state

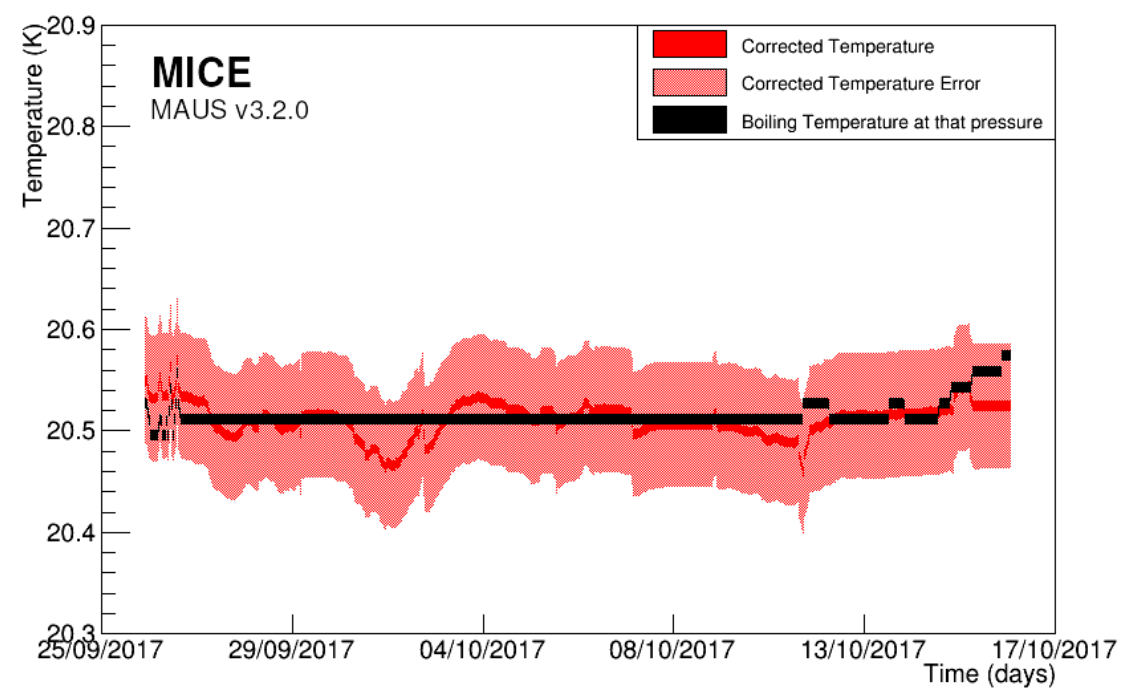

Figure 18. Average liquid hydrogen temperature recorded by the sensors during the steady state period. After applying all the correction factors the temperature remains at or close to the boiling point temperature.

Deflection of absorber vessel windows due to internal pressure. To minimise energy loss and Coulomb scattering by the absorber vessel, the window thickness was minimised. The liquid hydrogen circuit was pressurised above atmospheric pressure to prevent air ingress [41, 45]. The vessel was designed to withstand at least 2500 mbar internally. The internal pressure was limited by the 1.5 bar relief valve to atmosphere, whilst the vessel was surrounded by vacuum.

The pressure at which the absorber operated resulted in deflection of the absorber windows. These deflections were modelled using ANSYS [46], and the uncertainty in the window deflection derived from this model was $20 \%$. The model showed a linear dependence of the window deflection on pressure up to 2 Bar when the windows begin to yield. The pressure sensors were accurate to \pm 5 mbar $(0.25 \%$ of $2 \mathrm{Bar})$. At $(1085 \pm 5)$ mbar, the typical MICE operating pressure, this corresponded to a deflection of $(0.5374 \pm 0.1076) \mathrm{mm}$ (model uncertainty) $\pm 0.0022 \mathrm{~mm}$ (sensor uncertainty) at the centre of the absorber window.

Variation of the absorber vessel window thicknesses. On its passage through the absorber a muon would lose energy in the aluminium of the pair of hydrogen-containment windows, the two aluminium safety windows, and the liquid hydrogen itself. At the centre of the absorber, the total amount of aluminium the muon beam passed through was $(785 \pm 13) \mu \mathrm{m}$, producing a variance of $1.68 \%$. However, as the windows were thin, the effects on energy loss were negligible. A $200 \mathrm{MeV} / \mathrm{c}$ muon passing along the central axis of an empty absorber lost $0.345 \mathrm{MeV}$, introducing a $0.006 \mathrm{MeV}$ uncertainty on energy loss.

Total systematic uncertainty on energy loss. The principal contributions to the systematic uncertainty on energy loss in the liquid hydrogen absorber are: the uncertainty in the contraction of the absorber vessel, the uncertainty in the deflection of the hydrogen-containment windows due to internal pressure, and the uncertainty in the variation of the window thickness. The impact of the 
contraction of vessel and the deflection of the windows resulted in a reduction of the length of the vessel on axis of $(0.4 \pm 0.2) \mathrm{mm}$. The change in the combined thicknesses of the absorber windows on axis is $13 \mu \mathrm{m}$. The average temperature during the steady state period of the experiment when the pressure remained constant at $(1085 \pm 5)$ mbar is $(20.51 \pm 0.07) \mathrm{K}$ corresponding to a liquid hydrogen density of $(70.53 \pm 0.08) \mathrm{kg} / \mathrm{m}^{3}$.

During the MICE data taking, muon beams with nominal momenta of 140, 170, 200 and $240 \mathrm{MeV} / c$ were used. The energy loss and its uncertainty were calculated. The calculation used a central bore length of $(349.6 \pm 0.2) \mathrm{mm}$, a total window thickness of $(0.785 \pm 0.013) \mathrm{mm}$ and a liquid hydrogen density of $(70.53 \pm 0.08) \mathrm{kg} / \mathrm{m}^{3}$. For a $140 \mathrm{MeV} / \mathrm{c}$ muon this corresponds to an energy loss of $(10.88 \pm 0.02) \mathrm{MeV}$, while for a $200 \mathrm{MeV} / c$ muon particle this corresponds to an energy loss of $(10.44 \pm 0.02) \mathrm{MeV}$. For a muon travelling along the centre axis of the absorber the systematic uncertainty in the energy loss is $0.2 \%$.

\section{Summary and conclusions}

A complete set of particle detectors has permitted the full characterisation and study of the evolution of the phase space of a muon beam through a section of a cooling channel in the presence of liquid hydrogen and lithium hydride absorbers, leading to the first measurement of ionization cooling. The PID performance of the detectors is summarised in table 4 and table 5 and is fully compatible with the specification of the apparatus [47].

Table 4. Summary of the performance of the MICE PID detectors.

\begin{tabular}{|c|c|c|}
\hline Detector & Characteristic & Performance \\
\hline Time-of-Flight & time resolution & $0.10 \mathrm{~ns}$ \\
KLOE-Light & muon PID efficiency & $99 \%$ \\
Electron Muon Ranger & electron PID efficiency & $98.6 \%$ \\
\hline
\end{tabular}

Table 5. Summary of the MICE PID detector performance for different beam settings.

\begin{tabular}{|c|c|c|c|c|c|c|c|c|c|c|}
\hline \multirow{3}{*}{ Momentum } & \multicolumn{3}{|c|}{ KL efficiency } & \multicolumn{2}{|c|}{ EMR efficiency } & \multicolumn{5}{|c|}{ Track finding efficiency } \\
\hline & \multirow{2}{*}{ electrons } & \multirow{2}{*}{ muons } & \multirow{2}{*}{ pions } & \multirow{2}{*}{ electrons } & \multirow{2}{*}{ muons } & \multicolumn{2}{|c|}{$3 \mathrm{~mm}$} & \multicolumn{2}{|c|}{$6 \mathrm{~mm}$} & $10 \mathrm{~mm}$ \\
\hline & & & & & & US & DS & US & DS & US \\
\hline $140 \mathrm{MeV} / c$ & $95 \%$ & $97 \%$ & n.a. & $98 \%$ & $35 \%$ & & & $98 \%$ & $99 \%$ & $98 \% \quad 99 \%$ \\
\hline $170 \mathrm{MeV} / c$ & $95 \%$ & $99 \%$ & $89 \%$ & $99 \%$ & $99 \%$ & & & & & \\
\hline $200 \mathrm{MeV} / c$ & $94 \%$ & $99 \%$ & $95 \%$ & $100 \%$ & $99 \%$ & $99 \%$ & $96 \%$ & $99 \%$ & $96 \%$ & \\
\hline $240 \mathrm{MeV} / c$ & $96 \%$ & $99 \%$ & $97 \%$ & $99 \%$ & $99 \%$ & & & & & \\
\hline $300 \mathrm{MeV} / c$ & $95 \%$ & $99 \%$ & $98 \%$ & n.a. & $99 \%$ & & & & & \\
\hline
\end{tabular}

All the different elements of the MICE instrumentation have been used to characterise the beam and the measurement of the cooling performance for a different variety of beam momenta, emittance, and absorbers. The measurement of the physical properties of the liquid hydrogen 
absorber have been fully described here. The experiment has thus demonstrated a technique critical for a muon collider and a neutrino factory and brings those facilities one step closer.

\section{Acknowledgments}

The work described here was made possible by grants from the Department of Energy and National Science Foundation (U.S.A.), the Istituto Nazionale di Fisica Nucleare (Italy), the Science and Technology Facilities Council (U.K.), the European Community under the European Commission Framework Programme 7, the Japan Society for the Promotion of Science and the Swiss National Science Foundation, in the framework of the SCOPES programme. We gratefully acknowledge all sources of support. We acknowledge the use of Grid computing resources deployed and operated by GridPP in the U.K. [48]. We are also grateful to the staff of ISIS for the reliable operation of ISIS.

The MAUS software used to reconstruct and analyse the MICE data is available at [49].

\section{References}

[1] D.V. Neuffer and R.B. Palmer, A high-energy high-luminosity $\mu^{+}-\mu^{-}$collider, Conf. Proc. C 940627 (1995) 52.

[2] R.B. Palmer, Muon colliders, Rev. Accel. Sci. Tech. 7 (2014) 137.

[3] S. Geer, Neutrino beams from muon storage rings: characteristics and physics potential, Phys. Rev. D 57 (1998) 6989 [Erratum ibid. 59 (1999) 039903] [hep-ph/9712290].

[4] ISS Physics Working Group collaboration, Physics at a future neutrino factory and super-beam facility, Rept. Prog. Phys. 72 (2009) 106201 [arXiv: 0710.4947].

[5] M. Apollonio et al., Oscillation physics with a neutrino factory, hep-ph/0210192.

[6] S.Y. Lee, Accelerator physics, third edition, World Scientific, Singapore (2012).

[7] S. Schröder et al., First laser cooling of relativistic ions in a storage ring, Phys. Rev. Lett. 64 (1990) 2901.

[8] J.S. Hangst, M. Kristensen, J.S. Nielsen, O. Poulsen, J.P. Schiffer and P. Shi, Laser cooling of a stored ion beam to $1 \mathrm{mK}$, Phys. Rev. Lett. 67 (1991) 1238.

[9] P.J. Channell, Laser cooling of heavy-ion beams, J. Appl. Phys. 52 (1981) 3791.

[10] J. Marriner, Stochastic cooling overview, Nucl. Instrum. Meth. A 532 (2004) 11 [physics/0308044].

[11] V.V. Parkhomchuk and A.N. Skrinsky, Electron cooling: 35 years of development, Phys. Usp. 43 (2000) 433 [Usp. Fiz. Nauk 170 (2000) 473].

[12] A. Antognini et al., Demonstration of muon-beam transverse phase-space compression, Phys. Rev. Lett. 125 (2020) 164802 [arXiv: 2003. 11986].

[13] A.N. Skrinsky and V.V. Parkhomchuk, Cooling methods for beams of charged particles (in Russian), Sov. J. Part. Nucl. 12 (1981) 223 [Fiz. Elem. Chast. Atom. Yadra 12 (1981) 557].

[14] D. Neuffer, Principles and applications of muon cooling, Part. Accel. 14 (1983) 75.

[15] MICE collaboration, Demonstration of cooling by the Muon Ionization Cooling Experiment, Nature 578 (2020) 53 [arXiv: 1907.08562$].$

[16] M. Bonesini, The design of MICE TOF0 detector, MICE note 145, (2006), accessed 14 May 2021. 
[17] R. Bertoni et al., The construction and laboratory tests for MICE TOF0/1 detectors, MICE note 241, (2008), accessed 14 May 2021.

[18] MICE collaboration, The design and commissioning of the MICE upstream time-of-flight system, Nucl. Instrum. Meth. A 615 (2010) 14 [arXiv: 1001.4426].

[19] R. Bertoni et al., The construction of the MICE TOF2 detector, MICE note 286, (2010), accessed 14 May 2021.

[20] MICE collaboration, Pion contamination in the MICE muon beam, 2016 JINST 11 P03001 [arXiv: 1511.00556].

[21] MICE collaboration, The MICE muon beam on ISIS and the beam-line instrumentation of the Muon Ionization Cooling Experiment, 2012 JINST 7 P05009 [arXiv: 1203 .4089].

[22] MICE collaboration, Characterisation of the muon beams for the Muon Ionisation Cooling Experiment, Eur. Phys. J. C 73 (2013) 2582 [arXiv: 1306.1509].

[23] MICE collaboration, Electron-muon ranger: performance in the MICE muon beam, 2015 JINST 10 P12012 [arXiv: 1510.08306].

[24] MICE collaboration, First particle-by-particle measurement of emittance in the Muon Ionization Cooling Experiment, Eur. Phys. J. C 79 (2019) 257 [arXiv: 1810 .13224].

[25] M. Bonesini, R. Bertoni, A. de Bari and M. Rossella, Behaviour in magnetic fields of fast conventional and fine-mesh photomultipliers, Nucl. Instrum. Meth. A 693 (2012) 130 [arXiv: 1207.4909].

[26] M. Bonesini et al., The TOF1 local shielding, MICE note 455, (2015), accessed 14 May 2021.

[27] Y. Karadzhov et al., TOF detectors time calibration, MICE note 251, (2009), accessed 14 May 2021.

[28] L. Cremaldi, D.A. Sanders, P. Sonnek, D.J. Summers and J. Reidy Jr, A Cherenkov radiation detector with high density aerogels, IEEE Trans. Nucl. Sci. 56 (2009) 1475 [arXiv: 0905 . 3411].

[29] L. Cremaldi et al., Progress on Cherenkov reconstruction in MICE, MICE note 473, (2015), accessed 14 May 2021.

[30] F. Ambrosino et al., Calibration and performances of the KLOE calorimeter, Nucl. Instrum. Meth. A 598 (2009) 239.

[31] E. Overton, Studies and developments within the Muon Ionisation Cooling Experiment, Ph.D. thesis, Sheffield U., Sheffield, U.K. (2014).

[32] R. Asfandiyarov et al., MAUS: the MICE Analysis User Software, 2019 JINST 14 T04005 [arXiv: 1812 .02674].

[33] R. Asfandiyarov et al., The design and construction of the MICE electron-muon ranger, 2016 JINST 11 T10007 [arXiv: 1607.04955].

[34] F. Drielsma, Electron-muon ranger: hardware characterization, master's thesis, Geneva U., Geneva, Switzerland (2014) [arXiv: 1710.06946].

[35] F. Drielsma, Measurement of the increase in phase space density of a muon beam through ionization cooling, Ph.D. thesis, Geneva U., Geneva, Switzerland (2018)

[36] M. Ellis et al., The design, construction and performance of the MICE scintillating fibre trackers, Nucl. Instrum. Meth. A 659 (2011) 136 [arXiv: 1005. 3491].

[37] M. Petroff and M. Stapelbroek, Photon-counting solid-state photomultiplier, IEEE Trans. Nucl. Sci. 36 (1989) 158. 
[38] D0 collaboration, The upgraded D0 detector, Nucl. Instrum. Meth. A 565 (2006) 463 [physics/0507191].

[39] A. Dobbs et al., The reconstruction software for the MICE scintillating fibre trackers, 2016 JINST 11 T12001 [arXiv: 1610.05161].

[40] F. Drielsma, Beam-based detector alignment in the MICE muon beam line, arXiv: 1805. 06623.

[41] MICE collaboration, The liquid-hydrogen absorber for MICE, 2018 JINST 13 T09008 [arXiv: 1807.03019].

[42] Temperature measurement and control catalog, https://www.lakeshore.com/docs/default-source/product-downloads/lakeshoretc_l.pdf, accessed 2 October 2018.

[43] C. Brown et al., Systematic uncertainties in the liquid hydrogen absorber, MICE note 524, (2018), accessed 14 May 2021.

[44] G. Hardin, Aluminum 6061-T6 (UNS AA96061), https://www.nist.gov/mml/acmd/aluminum-6061-t6-uns-aa96061, accessed 3 October 2018.

[45] S. Ishimoto, S. Suzuki, M. Yoshida, M.A. Green, Y. Kuno and W. Lau, Liquid hydrogen absorber for MICE, Conf. Proc. C $\mathbf{1 0 0 5 2 3 ~ ( 2 0 1 0 ) ~} 421$.

[46] M. Green et al., Does one know the properties of a MICE solid or liquid absorber to better than 0.3 percent?, MICE note 155, (2006), accessed 14 May 2021.

[47] MICE collaboration, Proposal to the Rutherford Appleton Lab: an international Muon Ionization Cooling Experiment (MICE), MICE note 21, (2003), accessed 14 May 2021.

[48] D. Britton et al., GridPP: the U.K. grid for particle physics, Phil. Trans. Roy. Soc. A 367 (2009) 2447.

[49] MICE collaboration, Source code of MAUS - the MICE Analysis User Software, 10.17633/rd.brunel.8337542.v2, (2019). 